Infectious Diseases in Obstetrics and Gynecology 7:293-31 I (1999)

(C) 1999 Wiley-Liss, Inc.

\title{
European Society for Infectious Diseases in Obstetrics and Gynecology
}

\author{
7th European Conference of Infections \\ in Obstetrics and Gynecology
}

October 4-6, 1999

Istanbul, Turkey 
Prenatal Diagnosis of Fetal Cytomegalovirus (CMV) Infection

Wilczynski J., ${ }^{*}$ Nowakowska D., * Szaflik K, ** Paradowska E., ${ }^{* * *}$ Przepiörkievwicz M., ${ }^{* * *}$ Lesnikowski J.***

*Research Institute Polish Mother's Memorial Hospital, Department of Fetal-Maternal Medicine; **Research Institute Polish Mother's Memorial Hospital, Department of Ultrasonography, ${ }^{* * *}$ Center of Microbiology and Virology, Polish Academy of Sciences, Lodz, Poland

Introduction: The objectives were: 1 . to present our preliminary experience in sixteen pregnant women undergoing a prenatal evaluation to assess for fetal infection; 2 . to estimate the reliability of CMV-specific IgM as an indicator of fetal infection using polymerase chain reaction (PCR) for amplification of DNA. Patients: Sixteen patients were referred for prenatal diagnosis of suspected fetal cytomegalovirus infection (CMV). Two women had documented positive IgG and IgM(one with IgA positive) immunoglobulin titres in the first trimester of pregnancy and two in the second trimester. Twelve patients were evaluated because of abnormal ultrasonographic examinations under suspicion of fetal infection.

Methods: Mother's serum was assayed for the presence of CMV-specific IgM by an enzyme-linked immunoabsorbent assay (ELISA). Four methods of fetal diagnosis were employed involving a combination of amniocentesis, fetal blood sampling, prenatal paracentesis and thoracentesis. Six fetuses underwent fetal intervention at a median gestational age (range 28 to 30 weeks). Five out of sixteen cases had bilateral pleural effusions and hydrops. DNA was extracted from cultures of mother's blood and urine, amniotic fluid, fetal cord blood, pleural effusions and ascetic fluid. For the detection of CMV DNA in DNA preparations the nested-PCR test constructed in the Center of Microbiology and Virology PAS and PCR based TaKaRa (Japan) Human Cytomegalovirus infection was used.

Results: Out of twelve patients evaluated because of abnormal ultrasonographic examinations suspective fetal CMV infection serological methods (positive IgG and negative $(\mathrm{gM}$ ) in three cases DNA studies confirmed the presence of fetal CMV infection. Out of four patients with positive serological tests in one case with positive IgG, IgM and IgA DNA studies documented the presence of fetal infection and in three patients results suggested most likely a false-positive IgM.

Investigation of the Immune and Interferon Systems in Patients With Various HPV Type

Apolikhina Inna A., * Daragan Maria E., * Loginova Natalia S., ** Matveeva Natalia K., ** Sukhikh Gennady T.** *GAZPROM Policlinic, Moscow, Russia ul. Nametkina 16 **Research Center of Ob/Gyn \& Perinatology, Moscow, Russia ul. Academic Oparina 4

Introduction: Human papillomavirus (HPV) 16 and 18 has the most frequent causal contribution to the origin of cervical cancer. Since development of most cancers connect with an incompetence of immune and interferon (IFS) systems, the objective of the study was investigation of immune and IFS systems in patients with various HPV types.

Materials and Methods: We studied immune and IFS status in 117 patients with HPV detected by traditional and modern diagnostic technologies (PCR and DNA HPV Hy- brid Capture). The using of DNA HPV Hybrid Capture let us separate the patients into two groups: high-risk HPV (1) and low-risk HPV (2). The third group (control) consisted of 43 women without HPV infection. We have detected lymphocytic subpopulations composition peripheral blood by cytofluorimety methods with using monoclonal antibodies (Beckton Dickinson, USA).

Results: In the group with high-risk HPV the significant immune and IFN systems changes were detected in comparison to the group with low-risk HPV and among the controls. Statistic analysis of the lymphocytic subpopulations composition data shows significant differences in lymphocytic markers expression in both groups compared to controls. Amount of CD8+ lymphocytes increased in the high-risk HPV group and consequently $\mathrm{CD} 4+/ \mathrm{CD} 8+$ ratio decreased. Marker CD69+ was detected (after in vitro cell stimulation) in $68.5 \%$ cells in group (1) at that time, in group (2)and control patients it was $55.4 \%$ and $53.6 \%$ cells respectively. The indexes of $\alpha$-IFN decreased 4-6 times and those of $\gamma$-IFN decreased 6-8 times in comparison to control group. A dramatic elevation of serum and spontaneous IFN was found.

Conclusion: Diseases of the lower genital system caused by HPV develop in the presence of suppression of immune and interferon systems, reliable decrease of gammainterferon followed by an increase of serum interferon, and suppression of T-cellular chain activity which is expressed by an increase of CD8+ leukocytes and a decrease in CD4+/CD8 + ratio. This study leads to an individual choice of immunocorrectors for a patient in accordance with individual therapeutic approach, based on the individual hyperactivity.

Vaginal Microflora in Women Using OralAnticonception Gilbert GG Donders, Annie Vereecken, Alfons Dekeesmabcker, Bernard Spitz

Gasthuisberg University Hospital, Universiteit Leuven, and Laboratorium voor Klinische Pathologie, Antwerpen, Belgium

Introduction: A normal vaginal flora can be defined as a lactobacillus dominant ecosystem of microorganisms that prevent endogenous cohabitants and pathogens to profilerate. Materials: Women presenting in the vulvovaginitis clinic, Gasthuisberg University Hospital Leuven, Belgium.

Methods: Fresh vaginal fluid microscopy (lactobacillary grades), composite criteria according to Amsel (BV) vaginal $\mathrm{pH}$ and vaginal lactate in 113 oral anticonception (OAC) users versus 124 control women not using any anticonception ( 16 of whom had undergone tubal ligation), presenting in the vulvovaginitis clinic.

Results: A healthier flora was seen in the OAC users. Lactobacillary grade III (grossly abnormal) was present in $24 \%$ of OAC users, versus $33 \%$ in controls. Bacterial vaginosis (BV) and aerobic vaginitis (AV) were both present in $11 \%$ of OAC users and in respectively $15.4 \%$ and $18.5 \%$ $(P=0,058)$ of the controls. Lactate concentration was not different in both groups (mean $114 \mathrm{mg} / \mathrm{dl}$ in OAC users vs 95 $\mathrm{mg} / \mathrm{dl}$ in controls). Mean $\mathrm{pH}$ was lower in OAC users $(P=0.05)$. OAC users were younger than the controls $(P<0.001)$.

Conclusion: OAC use does not cause deterioration of the vaginal lactobacillary flora and may rather protect women against $\mathrm{BV}$ and $\mathrm{AV}$. 
Apparent Failure of One Injection of Benzathine Penicillin $\mathbf{G}$ for Syphilis During Pregnancy in HIV Seronegative African Women

Donders GGG, ${ }^{*}$ Desmyter J., ${ }^{*}$ Hooft P,* Dewt GH**

*Katholieke Universiteit, Leuven, Belgium; **Universiteit van Pretoria, South Africa

Objectives: Is one injection of 2.4 million units of benzathine penicillin $\mathrm{G}$ sufficient treatment for early syphilis during pregnancy (CDC guidelines 1993)?

Study Design: Outcome of pregnancy was prospectively analysed after 0 to 3 weekly intramuscular injections of benzathine penicillin $G$ in 180 of 212 HIV seronegative black urban women with syphilis in Pretoria, South Africa.

Results: One hundred and eight women receiving two or three weekly intragluteal injections of benzathine penicillin $\mathrm{G}$ had a favourable pregnancy outcome. However, after only one injection, lower birth weight, increased immaturity, prematurity, and total preterm birth rate resulted. Treponemicidal coverage of 3 weeks or less resulted in decreased birth weight ( 2748 vs. $3130 \mathrm{~g}, P=0.004$ ) when compared to treponemicidal coverage lasting longer than three weeks. Also the relative risk for prematurity (OR 8.5, cl95 2.5-28). perinatal mortality (OR 20.5, c195 2.3-1 84) and congenital syphilis (RR 2.0, c195;0.6-6.8) were increased when coverage was less then 3 weeks. These results were comparable to when no treatment was given. The majority of incompletely treated women delivered at less than 4 weeks after they received their injection. These also had the worst neonatal outcome. Impaired outcome due to short treatment clustered in early attainders of prenatal care and when the initial RPR titer was higher than 16.

Conclusion: One intramuscular injection of 2.4 million units benzathine penicillin $\mathrm{G}$ or treponemicidal concentrations lasting 3 weeks or less are not sufficient therapy for pregnant women with syphilis. Two injections of 2.4 million units benzathine penicillin at least one week apart are recommended.

Cytometric DNA Analysis of Condyloma Acuminatum With Two Different Treatment Approaches

Yorganci A,* Dünder I,** Ortac F,** Tezcan S**

*Ankara Numune Hospital, Department of Obstetrics and Gynecology, Ankara, Turkey

**Ankara University, Faculty of Medicine, Department of Obstetrics and Gynecology, Ankara, Turkey

Condyloma acuminatum is a sexually transmitted disease with high recurrence rates. The aim of this study was to predict the recurrence of this disease before using two different treatment modalities by flow cytometric DNA analysis.

Twenty-seven patients with vulvar condyloma acuminata were randomly divided into 2 groups. From these, 16 patients were treated with $\mathrm{CO}_{2}$ laser and 11 patients were treated with intralesional interferon. Before treatment, each patient was evaluated with flow cytometry to determine the presence of aneuploidy, DNA index, and $S$ phase. The features of flow cytometry of the recurrent cases were analysed.

The recurrence rate of patients treated with $\mathrm{CO}_{2}$ laser and interferon treatment were $25 \%(4 / 16)$ and $9 \%(1 / 11)$, respectively. Flow cytometric DNA analysis revealed $18.5 \%$ diploidy and $81 \%$ aneuploidy in 2 patient groups. The presence of aneuploidy, DNA index, and S phase were not statistically different in both treatment modalities and in recurrent and nonrecurrent patients.
The results of this study showed that the prediction of the recurrence of condyloma acuminata can not be possible with flow cytometric DNA analysis.

Analysis of the Selected Intraspecies and Species Features of Strains From Vaginal Ontocenosis of Women With Diabetes Mellitus

D.Nowakowska,* J. Wilczynski,* A. Kurnatowska**

*Institute Polish Mother's Memorial Hospital, ${ }^{* *}$ Chair of Medical Biology and Parasitology, Medical University of Lodz, Poland

Introduction: The objective of the study was the analysis of the fermentation properties and the ability of carbon assimilation from 19 chemical compounds by fungi strains isolated from vaginal ontocenosis of women with diabetes mellitus. Materials and Methods: The fungal strains were isolated from vaginal ontocenosis of 72 women with diabetes mellitus whose mean glucose values did not exceeded $5.27 \mathrm{mmol} /$ L (95 mg/dl). 30 morphological (macroscopic and microscopic) features of cultures (vegetative cells, blastospores, pseudohyphae), formation of ascospores (Gorodcov's medium) and biochemical features of each of 63 axenic strains were described for species identification. The strains were identified using API $20 \mathrm{C}$ and API 20C AUX tests according to the principle of numerical identification (Analytic Profile Index, bioMerieux, Lyon 1990).

Results: Out of 63 fungal strains, 53 were presented by C.albicans, 4 by C.glabrata, 3 by C.tropicalis, 1 by C.parapsilosis and 2 by S.cerevisae. There were 4 codes identified for C.albicans $(2576174-76.6 \%, 657174-1.6 \%$, $2776174-4.8 \%, 2572174-2.7 \%), 2$ codes for C.tropicalis (25763750-1.6\%, 2576177-2.7\%) and 1 code separately for C.glabrata (2000040-6.4\%), C.parapsilosis (6756135-1.6\%) and S.cerevisae (2040070-2.7\%). The chemical compounds showing taxonomic value for subspecific discrimination of species was glycerol, xylitol, 2-keto-D-gluconian and Lmethylo-D-glucoside.

Conclusion: 1 . In spite of the repeated typical morphological characteristics (macroscopic and microscopic) of $C$ albicans, there are significant biochemical intraspecies differences. 2. Intraspecies biochemical differentiation may explain the potential pathogenic abilities of the fungal strains isolated from vaginal ontocenosis of pregnant women with diabetes mellitus.

Correlation Between Clinical Symptoms of Candidosis and Selected Morphological and Biochemical Features of Candida Strains Isolated From Ontocenoses of Vagina and Oral Cavity of Pregnant Diabetic Women

D.Nowakowska, J. Wilczynski, ${ }^{*}$ A. Kurnatowska**

*Institute Polish Mother's Memorial Hospital, **Chair of Medical Biology and Parasitology, Medical University of Lodz, Poland

Abstract: The objective of the study was to evaluate the correlation between clinical data and 50 characteristics of Candida strains isolated from two ontocenoses of pregnant diabetic women with different types of diabetes mellitus. The relationship between clinical symptoms and different fungal features was searched according to their own scheme. Data were collected in a special computer program. The fungal strains were isolated from 100 women with pregestational 
diabetes mellitus (PGDM) or gestational diabetes mellitus (GDM:GDM-1,GDM-2). Clinical evaluation of the 10 signs and symptoms of candidosis was performed. The control group covered 100 pregnant women without diabetes mellitus. The characteristics of axenic strains were assessed owing to their own methods and unified tests (bioMerieux: API2O C, API2O AUX, API 20 ZYM). Correlation coefficient (r) was used to measure the relationship between two variables. The prevalence of fungal strains in diabetic pregnant women was $30-50$ percent. In the control group candidosis occured in 20 percent of women. The highest correlation coeffcient ( $r$ ) related to bifocal infections of Candida. In the group of diabetic pregnant women there was a strong correlation between presence of fungi in vagina ( $\mathrm{r}$ from +0.455 to +0.720 ) and the symptoms, i. e., pruritus, swelling, redness, burning, abnormal discharge, and general diagnosis of colpitis. There are differences in biochemical characteristics of fungal strains isolated from diabetic and nondiabetic women. The correlation for the clinical symptoms of genital infections refer mainly to the high activity of several hydrolases, i.e., esterase (e3), valine arylamidase (e7), naphtol-AS-BI-phosphohydrolase (e 12), a-glucosidase (e 16). Additional studies are needed to define the relationship between clinical data and Candida strains isolated from different ontocenoses of pregnant women with diabetes.

\section{Pathogenesis of Bacterial Vaginitis}

Donders GGG, * Bosmans E,** Dekeersmaecker A,** Vereecken A,** Van Bulck B,*** Spitz B*

${ }^{*}$ Gasthusberg Hospital, Katholieke Universiteit Leuven, **Laboratory of Clinical Pathology, Antwerp, ***Erasmus Hospital, Antwerp, Belgium

Aim: To determine the relationships between microscopic findings on wet mounts and results of vaginal culture, lactate and succinate content of the vagina and selected cytokinesis. Methods: From 631 women presenting at an obstetrics/ gynaecology outpatient clinic vaginal fluid was obtained for wet mount, $\mathrm{pH}$ measurement, culture and measurements of lactate, succinate, IL-1-beta, IL-8, leukemia inhibitory factor (LIF) and interleukin-1-receptor antagonist (IL-1-RA).

Results: Increasing disturbance of the lactobacillary flora (lactobacillary grade (LBG) I, IIa, IIb, III) was highly correlated with the presence of Garderella vaginalis, Trichomonas vaginalis, enterococci, group B streptococci and Escherichia coli. Vaginal pH, IL-8 and IL1 beta increased linearly with increasing LBG, while lactate concentrations and the presence of epithelial cell lysis decreased. A similar pattern of associations with increasing leukocyte count was clear, but, in addition, there was an increase in LIF concentration. The IL-1-betal/IL-1-RA ratio remained stable, except when vaginal leukocytosis increased. In its severest form, with more than 10 leukocytes per epithelial cell present, a decompensation of the vaginal flora with collapsing IL-1-beta and IL-1RA concentrations was seen, but with a concurrent sharp increase in LIF concentration. This pattern was completely different from the course of the cytokine concentrations associated with an LBG increase.

Discussion: There was a remarkably linear increase in cytokines when either leukocytosis or LBG became more severe. When leukocytosis was extreme, IL-1-beta was no longer produced, but LIF became more severe. We speculate that in extreme inflammation the body tries to limit the damage that can be done by exaggerated cytokine production.
Peroxide Oxidation of Proteins (Oxidizing Modification of Protein) in Pregnancy Taking Its Physiological Course Ivanov II,

Crimea State Medical University, named after S.I.Gergievsky (chair of Obstetrics and Gynaecology No:2, chief - assistance - Professor Yu.P.Pasechnikov).

The aim of the work was to study the state of peroxide oxidation of proteins in healthy pregnant women. 20 healthy pregnant women aged 18 to 25 within $34-40$ weeks of gestation were under observation. As a result of investigations it has been established that in healthy pregnant women, compared to healthy nonpregnant women, there is observed lowering of the level of processes of protein oxidation, excluding the rate of oxidizing processes, i.e., the level of metabolic processes is increased.

\section{Unasyn Prophylaxis at Obstetrical Operations} Bagdany S, Szathary Gy, Zsolnai B, Plaszko Zs Weiss Manfred Postgraduate Hospital, Department of Obstetrics and Gynaecology, Budapest, Hungary

There is different opinion about antibiotic prophylaxis in obstetrics and gynaecology. Some doctors suggest the Metronidazole-infusion at caesarean sections, but many obstetricians use other preparations. We had tried Metronidazole infusion previously in the Postgraduate Medical School of Hungary, but in the past two years we began to give Unasyn for prophylaxis. There was given $2 \mathrm{~g}$ Unasyn once before the operations. The results showed that the number of complications decreased from 12 to $7.8 \%$. It is our opinion that Unasyn can be used with good results for prophylaxis.

Sensitivity to Antibiotics of Group B Streptococcus Isolated From Vaginal Specimens of Pregnant Women Shopova E, Markova $\mathrm{M}^{* *}$

Microbiological Laboratory, University Hospital of Obstetrics and Gynecology. **Chair of Clinical Laboratory and Immunology, Sofia, Bulgaria

Over a period of 20 months we investigated 1366 vaginal specimens from pregnant women for GBS (group B streptococci carrier in three microscopic patterns, evaluated by Nugent score system). More frequently we isolated GBS in gr.I (4-6 score) - 20.8\%,(when Lactobacillus Spp. is missing $(67.4 \%)$, without or associated with GBS strains showed sensitivity to ampicillin and carbenicillin. Good sensitivity was found to cefazolin $(92.4 \%)$ and to cefuroxim $(94.2 \%)$. The strains showed $13.7 \%$ resistance to erythromicin and $4.1 \%$ to clindamycin.

\section{Infectious Factor in Premature Labour and Neonatal Fates}

Was K, Reron A, Rzepecka-Weglarz B, Wiechec M, TrojnarPodlesny M.

Department of Obstetrics and Gynecology, Jagiellonian University Hospital, Cracow, Poland.

Introduction: Although the frequency of premature labour in highly developed countries is established in only $6-8 \%$ of all labours, it is the cause of perinatal mortality in about $75 \%$. It is known that the beginning of the labour is a very complicated process, involving both mother and fetus, but 
one of the most important factors to be regarded is the infectious factor. Bacteria and their endotoxins induce increased prostaglandin synthesis, which stimulate uterine contracting activity.

Materials, Methods and Results: Between 1997-01-01 and 1999-07-31 in the Septic Ward of the Department of Obstetrics and Gynecology, Jagiellonian University Hospital in Cracow, 727 labours took place. 151 of all labours were premature $(20.7 \%)$. Premature rupture of membranes was identified in 38 pregnant women (5.2\%). At the time of admission on the ward the dilation of the external os was as follows: no dilatation in 78 pregnant women $(50.03 \%)$, less than $1 \mathrm{~cm}, 42(27.81 \%)$ from 1 to $3 \mathrm{~cm}, 19(12.58 \%)$, from 3 to $5 \mathrm{~cm}$, $8(5.29 \%)$, and more than $5 \mathrm{~cm}, 6(3,97 \%)$. In spite of tocolitic management (Partutisten, magnesium sulphate), between 24 and 37 weeks of gestation, 151 women laboured., in which in 87 cases $(57,61 \%)$ it was spontaneous labour, in 20 $(13,24 \%)$, pharmocologically supported vaginal delivery, and manual fetal extraction procedure in 1 case $(0.0062 \%) .113$ neonates was born alive $(74.83 \%)$, in which 9 had congenital defects $(5.96 \%)$ and 38 were stillborn $(25.16 \%)$. The neonates' estimation by Apgar scale was as follows: $8-10$ points, 55 neonates $(48,68 \%), 5-7$ points, $24(21,24 \%)$, less than 5 points, $34(30.10 \%)$.

Conclusion: Premature labour is associated with high rate of perinatal mortality and congenital defects.

\section{Evaluation of Thyroid Functions In Pregnant Women} Kesim M., Yalçin A., Karlik I., Kaya I., Iiili T.

Iiili Etfal Teaching and Research Hospital, Third Gynecology and Obstetric Clinic, Istanbul, Turkey

Objective: In our study, held in Iiili Etfal Teaching and Research Hospital, Third Gynecology and Obstetric Clinic, we aimed to evaluate the thyroid functions in different periods of pregnancy, disorders of thyroid functions found during pregnancy and their effects on maternal-fetal morbidity and mortality. We also aimed to evaluate the goiter cases during pregnancy.

Materials and Methods: We assessed 41 pregnant women in the first trimester and 118 in the third trimester. We also evaluated the thyroid function results of 150 women in reproductive period that applied to our hospital biochemistry department. Serum T3, T4, FT4 and TSH values were measured for all pregnant and nonpregnant women.

Results: First trimester FT4 values were significantly higher than the values of third trimester and nonpregnant women $(P<0.001)$. The progressive increase in T3 and T4 values during pregnancy were significant when compared with nonpregnant and first trimester values $(P<0.001)$. When we evaluated the 105 women in active labor the incidence of complications (preeclampsia and IUGR) was significantly higher in women with disorder of thyroid functions $(P<0.05$ and $P<0.01$ respectively). We found 33 goiter cases among 159 pregnant women. Conclusion: Changes in thyroid functions during pregnancy were consistent with the findings of other authors. We have also shown that the women with thyroid function disorders were under serious pregnancy risk (especialty preeclampsia and IUGR) and under the risk of poor neonatal outcome. The frequency of thyroid enlargement-goiter cases probably indicates the deficiency of iodine intake that increases during pregnancy. Thus, it would be appropriate to evaluate all goiter cases before and during the pregnancy period and to start the necessary treatment and supplementation.

\section{An Ovarian Pregnancy Case}

Kesim M,* Kaya I., ** Turgut S.," Karlik I.,* Yalçin A., Erdemir M., * Yilmaz B.**

*Iiili Etfal Hospital Third Obstetrics and Gynecology Clinic, Istanbul, Turkey

**Iiili Etfal Hospital Pathology Clinic, Istanbul, Turkey Ovarian pregnancy is a rare form of ectopic pregnancies. Among all the pregnancies that have attended in our outpatient clinic, the ectopic pregnancy ratio was $1 / 8000$ with one ovarian case $(1.25 \%)$ in a total of 80 ectopic pregnancies diagnosed. The differential diagnoses of ovarian pregnancy is difficult, the symptoms and signs are like other ectopic pregnancies. C. luteum hematoma is seen frequently. The diagnosis is made mostly during laparotomy and even after the operation during the pathological investigation. In the medical history of this ovarian pregnancy case, 4 pregnancies, one delivery (6 years ago, cesarean section), right salphingectomy because of ectopic pregnancy 2 years previously, and a history of PID were seen. There was no usage of IUD. In the literature it is seen that, unlike tubal pregnancies, ovarian pregnancy usually does not have any relation to PID and infertility and the most important risk factor for ovarian pregnancy is IUD usage. In contrast, in our ovarian pregnancy case there was no usage of IUD but a history of PID was seen. Since ovarian pregnancy is a rare form of ectopic pregnancies, we aimed to present this ovarian pregnancy case seen in our clinic.

The Bacterial Flora of the Vagina in Healthy Turkish Women and in Turkish Women With Bacterial Vaginosis Mamal Torun $\mathrm{M},{ }^{*}$ Bahar $\mathrm{H},{ }^{*}$ Öçer $\mathrm{F}$.**

Istanbul University, Medical Faculty of Cerrahpaia, *Department of Microbiology and Clinical Microbiology, ${ }^{* *}$ Department of Obstetrics and Gynecology, Istanbul, Turkey The aim of this study was to determine the bacterial flora of the vagina in healthy women (HW) and in women with bacterial vaginosis(BV). The vaginal samples of $100 \mathrm{HW}$ and samples of 100 women that were determined to have BV by clinical symptoms shown in microscopic exams of their Gramstained vaginal smears were studied. The facultative microorganisms and the anaerobic microorganisms were isolated and identified. The results were confirmed by API and SCEPTOR panels. The number of each microorganism was evaluated and for hypothesis testing the Mantel Haenszel test for linear association of ordinal data was used. The value $<0.05$ was considered statistically significant.

The isolation rate of facultative bacteria and anaerobic bacteria which grew in a high concentration and which showed a significant difference between the two flora (HW and BV) with a $P$ value $<0.01$ was as follows. Gardnerella vaginalis $75 \%$ in BV, $25 \%$ in HW, Mycoplasma hominis $71 \%$ in BV, $25 \%$ in HW, Ureaplasma urealyticum $84 \%$ in BV, $33 \%$ in HW, viridans streptococci $78 \%$ in BV $32 \%$ in HW and Lactobacillus sp $0 \%$ in BV, $53 \%$ in HW, Prevotella bivia $85 \%$ in BV, $8 \%$ in HW, P.disiens $83 \%$ in BV, $5 \%$ in HW, Fusobacterium nucleatum $69 \%$ in $\mathrm{BV}, 0 \%$ in $\mathrm{HW}$, Peptostreptococcus prevotii $68 \%$ in BV, $17 \%$ in HW, P.asaccharolyticus $66 \%$ in BV, $10 \%$ in HW, P.magnus $79 \%$ in BV, $0 \%$ in HW, Mobiluncus curtisö $14 \%$ in BV, $0 \%$ in $\mathrm{HW}$, M.mulieri $11 \%$ in BV and $0 \%$ in HW.

As a result we think that because of the recognized role of the endogenous vaginal flora in complications of pregnancy and infections of the upper genital tract it is important to 
gain a better understanding of the composition of this system in our population.

\section{Laparoscopic Diagnosis and Therapy of Salpingitis}

Udo B. Hoyme, Ute Mahnert

Department of Gynaecology and Obstetrics, Klinikum Erfurt, Germany

Objectives: Salpingitis has a complex etioiogy dominated by Chlamydia trachomatis $(C t)$ and by Neisseria gonorrhoeae $(\mathrm{Ng})$, however, the particular pathogen is difficult to identify, even in laparoscopically obtained specimens, since results from the lower genital tract do not always correlate with those from tubal swab samples. Several techniques have been described, including one invented in 1980 using a specially designed swab instrument directly inserted through the working channel of the laparoscope.

Study design: The microbiological results of tubal swab specimens are compared with those from urethral and cervical swabs of patients with laparoscopically diagnosed salpingitis. $C t$ was identified by means of PCR and/or LCR, $\mathrm{Ng}$ (and other bacteria) by standard cultures.

Results: Between 9/93 and 12/99 141 out of 321 patients with suspected PID were laparoscopically diagnosed to suffer of acute salpingitis due to the following bacteria:

\begin{tabular}{lcccc}
\hline & & $\begin{array}{l}\text { Brihmer } \\
\text { '89 }\end{array}$ & $\begin{array}{l}\text { Brunham } \\
\text { '88 }\end{array}$ & $\begin{array}{l}\text { Hoyme } \\
\text { '88 }\end{array}$ \\
\hline$N$ & 141 & 64 & 36 & 71 \\
C.trachomatis & & & & \\
Cervix & 4 & 36 & 4 & 11 \\
Urethra & 4 & n.d. & n.d. & 10 \\
Tubes & 31 & 12 & 2 & 8 \\
Tubes only & 27 & 0 & 1 & 3 \\
All & 35 & 36 & 5 & 17 \\
N.gonorrhoeae & & & & \\
Cervix & 0 & 15 & 18 & 5 \\
Urethra & 0 & 2 & 6 & 0 \\
Tubes & 1 & 0 & 0 & 0 \\
All & 1 & 15 & 18 & 5 \\
\hline
\end{tabular}

Conclusion: Besides the multitude of bacterial species involved, the difficulty in gaining access to the fallopian tubes is the major obstacle in microbiologic evaluation of salpingitis. However, a large proportion of $C t$ positive cases would be missed if laparoscopy was not performed.

Management of Uncomplicated (Postoperative) Urinary Tract Infections

Udo B. Hoyme

Department of Gynaecology and Obstetrics, Klinikum Erfurt, Germany

Cystitis or lower urinary tract infection (UTI) is estimated to occur at least once in $20 \%$ of all women in their lifetime. It increases from about $5 \%$ incidence in the reproductive age group to about $10 \%$ by the early postmenopausal years. UTI in the gynecological patient is also associated with medical, surgical and gynecological problems. The prevalence of infection is reported to be 15 to $25 \%$ in women with uterovaginal prolapse, 10 to $100 \%$ in patients with cervical carcinoma or 80 to $100 \%$ after surgery with catheterization and up to
$30 \%$ without catheterization. The prevalence of postoperative bacteriuria is correlated with the procedure performed, with vaginal and periurethral traumatization as well as with duration of catheter placement.

The spectrum of pathogens in UTI is Escherichia coli $76 \%$, Proteus 2\%, Pseudamonas 1\%, Klebsiella 1\%, Staphylococcus spp. 7\%, Streptococcus/enterococcus spp $12 \%$. For treatment, short-term and single-dose regimens are used in patients with uncomplicated or postoperative UTI since these infections involve only the superficial mucosal epithelium layers. The indications are as follows: Symptoms for less than one week, reproductive age women, women with iatrogenic infection, exclusion of predisposing disorders, no anatomical abnormalities, no diabetes, opportunity of clinical control, reliable patient.

In our own prospectively randomized studies we treated women with postoperative UTI by nitrofurantoin, trimethoprim/sulfamethoxazole and ciprofloxacin for two days or by single dose in comparison to established conventional therapy regimen: The efficacy of single-dose administration of $480 / 2400 \mathrm{mg}$ trimethoprim/sulfamethoxazole $(85 \%, n=82$ vs. $92 \%, n=83$; n.s., $\times 2$-test $)$ and $250 \mathrm{mg}$ ciprofloxacin $(94 \%, n=62$ vs, $95 \%, n=65 ;$ n.s. $)$ was as good as traditional policy. However, cure rates following two days of nitrofurantoin were demonstrated to be inferior (normal $69 \%, \mathrm{n}=67$ vs. $86 \%, \mathrm{n}=65 ; P<0,05$; macrocrystals $73 \%$, $\mathrm{n}=91$ vs. $85 \%, \mathrm{n}=99 ; P<0.05$ ), This is likely explained by the poor tissue penetration of this antimicrobial substance. In another prospectively randomized study, ciprofloxacin given to outpatients with uncomplicated UTI as $250 \mathrm{mg}$ single dose versus $250 \mathrm{mg}$ b.i.d. for 3 days led to cure rates of $18 \%$ and $91 \%(P<0.05)$. The high failure rate in the single dose group was mainly due to persistence of $E$. coli and reinfection with enterococci.

The benefits of short-term/single dose therapy of UTI are as follows: efficacy, excellent compliance, few side effects, low cost, limited emergence of resistant bacteria, identification of patients at risk by treatment failure.

\section{Reduction of Prematurity by pH-Screening-The '98 State}

Udo B. Hoyme,* A. Grosch,* V.M. Roemer,** E. Saling*** *Department of Gynaecology and Obstetrics, Klinikum Erfurt, **Department of Gynaecology and Obstetrics, Klinikum Detmold, ***Institute of Perinatal Medicine, Berlin, Germany

Objectives: Bacterial vaginosis (BV) increases the relative risk of prematurity. In these cases the preterm birth rate can be reduced by early intervention with antimicrobial substances such as clindamycin.

Study design: Women seeking prenatal care in one of 29 outpatient offices in the city beginning $10 / 96$ were informed and requested to practice self-measurement of the vaginal $\mathrm{pH}$ every three days in order to screen for BV: Care Plan $\mathrm{VpH}$ gloves (Selfcare, Oberhachingen) were used in identifying patients at risk by means of a $\mathrm{pH}>4.7$. These women were instructed to see their physician immediately to have BV possibly confirmed and treated with clindamycin cream (Sobelin, Upjohn, Erlangen) i.vag. initiated, otherwise lactobacilli $10^{7}$ estrioil $0.03 \mathrm{mg}$ (Gynoflor, Nourypharma, OberschleiBheim) were administered i.vag. for 6 days. Patients of 18 not participating local obstetricians and woman not consenting to participate in the study served as controls. 
Results: Up to $12 / 98,73$ of 381 women in the intervention group were identified with a $\mathrm{pH}>4.4$. 58 were treated with lactobacilli, 24 additionally with clindamycin cream because of BV and 3 had no therapy. In this ongoing study the prematurity rate was $8.1 \%$ in the intervention group vs. $12.3 \%$ in controls $(\mathrm{n}=2.341 ; P<.05) .0 .3$ vs. $3.3 \%$ of the neonates were born at $<32$ gestational weeks $(P<0.01)$. PROM was registered in $22.8 \%$ vs. $30.8 \%(P<0.01)$ respectively.

Conclusion: Self-measurement of vaginal $\mathrm{pH}$ at close intervals according to Saling seems to identify women at risk for prematurity. Earliest possible intervention either with lactobacilii or in case of confirmed BV with clindamycin appears to result in a reduction of preterm birth and in particular of newborns less than 32 weeks.

\section{A Case of Septic Shock, Resulting in Death After Opera- tive Delivery \\ Baigül Alin, Kavak Zehra, Pekin Sakip, Pekin Tanju}

Marmara University Hospital, Department of Obstetrics and Gynecology. Istanbul, Turkey

Septic shock is a life threatening disorder which can be associated with obstetric conditions. Successful management is of ultimate importance and depends on early identification and aggressive treatment. We report here a young woman who has developed septic shock after operative delivery in week 32 of pregnancy. Clinical features, treatment modalities, and prognosis of this high mortality rate disorder are presented and discussed.

Case Report: A 24-year-old woman, gravida 1, para 1, was referred to our clinic in a confused state and immediately admitted to our emergency service. She had eclampsia antenatally. Termination of pregnancy with induction of the labor following vacuum extraction had been applied in gestational week 32 of pregnancy. One day after delivery, her clinical and laboratory parameters worsened and she was referred to our clinic. After a thorough physical examination and laboratory evaluation, the patient was diagnosed with sepsis and disseminated intravascular coagulation. After blood and urine cultures were taken, aggressive management included volume repletion, antibiotics, and positive inotropic therapy. As she had persistent fever and unimproved laboratory values despite these therapies for 72 hours, the uterus and ovaries were thought to be the source of sepsis, total abdominal hysterectomy and bilateral salphingooopherectomy was performed. Neither clinical nor laboratory parameters improved and on the 16th postoperative day, the patient died of gram negative septicemia.

\footnotetext{
A Case of Septic Shock Developing Rapidly After Elective First Trimester Curettage

Baigül Alin, Durmuioilu Fatih, Tufan Ergün

Marmara University Hospital, Department of Gynaecology and Obstetrics. Istanbul, Turkey

Septic shock is a life threatening disorder that can be associated with obstetric conditions. Successful management is of ultimate importance and depends on early identification and aggressive treatment. We report here a young woman who developed septic shock rapidly after an elective first trimester abortion. Clinical features, treatment modalities, and prognosis of this high mortality rate disorder are presented and discussed.
}

Case Report: A 27-year-old woman, gravida 2, para 1, was admitted to our emergency service with the complaint of nausea, vomiting, and diarrhea which had started one to two hours after elective abortion that was performed earlier that day. She had fever of $38.5^{\circ} \mathrm{C}$, mild tacypnea and mild dyspnea. After a thorough physical examination and laboratory evaluation it was found that she had mild suprapubic tenderness, prolonged prothrombin time and elevated liver enzymes, fibrin degradation products and low platelet count. Her arterial blood gas measurement showed hypoxemia. The patient was diagnosed with sepsis and disseminated intravascular coagulation and admitted to the intensive care unit of our hospital. After blood and urine cultures were taken, aggressive management included volume repletion, antibiotics and positive inotropic therapy. Since the patient had a persistent fever and unimproved laboratory values despite these therapies for 72 hours, the uterus and ovaries were thought to be the source of sepsis and total abdominal hysterectomy and bilateral salphingooopherectomy was performed. Four days after the operation, all the clinical and laboratory parameters were improved and on the postoperative tenth day she was discharged from hospital with oral antibiotic therapy and estrogen replacement therapy.

The Prevalence of Urinary Infections in Women With Intrauterine Devices(IUD)

Cüce M,Yaiceken S, Benli Z, Arici A, Acarlar E, Ündar G, Ertopçu K, Özelmas I,Timoçin A.

Dept. of Family Planning, Aegean Social Insurance Inst., Obstetric \& Gynecology Hospital, Izmir, Turkey

Introduction: IUD is a reversible and the most effective contraception method used in Turkey. $18.8 \%$ of women in the reproductive period use IUDs.

Materials and Methods: 30 women admitted to the Clinic of Family Planning between the dates 3-14 August, 1999 for IUD controls without any complaint of bleeding were included in the study. Urine cultures were obtained by Nelaton catheter. The specimens were transferred to the Bloody and EMB agar. Antibiograms were prepared according to the Kirby-Bauer diffusion method. The direct microscopy of vaginal discharge was evaluated by saline and potassium hydroxide solution.

Results: In four of 30 cases urine cultures were positive $(13 \%)$. The bacteria in question were determined to be Klebsiella spp., Enterobacter spp., Coagulase negative staphylococcus and $E$. coli. Cases with positive cultures complained of inguinal pain. In only two of the cases dysuria and pollakuria were noted. There was no clinical complaint in five of 26 cases with negative cultures. In 14 of these cases inguinal pain, in 12 dysuria, in 1 pollakuria and in 10 nocturia were recorded.

Conclusion: The prevalence of urinary infection in IUD users is no different than the normal population.

\section{Policresulen in the Therapy of Recidivant Colpitis}

Kostadinov G.

Ob\& Gyn Center "Dr. Kelesov" Kumanovo, Macedonia

Introduction: The objective of the study is to fortify subjective and clinical improvement of the condition in the patients with recidivant colpitis, after the treatment with Policresulen vaginal suppositories, and to fortify the influence of the therapy in the finding of "satisfactory but 
limited with inflammation" PAP, which is very frequent in such patients.

Materials and Methods: Fifty-seven patients in the therapy group and 44 patients in the control group with symptoms and clinical findings of recidivant colpitis were included in this study. All patients were of reproductive age and HIV negative. Protocol for clinical assessment and questionnaire with "Discomfort score system" (0-3 Insignificant discomfort, 4-6 Moderate discomfort, 7-9 Hard discomfort) was used. Ten days treatment with Albothyl (Policresulen $90 \mathrm{mg}$, BYC GULDEN), with control of symptoms and clinical picture after 3 weeks was performed. PAP was reviewed before and three months after the treatment.

Results: Table 1. Distribution of a type of recidivant colpitis and mean discomfort scores (MDS) before treatment in the therapy and control groups:

\begin{tabular}{lcccrc}
\hline & \multicolumn{2}{c}{ Therapy group } & \multicolumn{3}{c}{ Control group } \\
No & $\%$ & MDS & No & $\%$ & MDS \\
\hline Bacterial colpitis & 25 & 44 & 7.1 & 19 & 43 \\
C. trichomonas & 20 & 35 & 8.4 & 16 & 36 \\
Candida colpitis & 12 & 21 & 7.0 & 9 & 21 \\
Total & 57 & 100 & & 44 & 100 \\
\hline
\end{tabular}

There were overall $19(43 \%)$ findings of "satisfactory but limited with inflammation" in previous PAP in the therapy group.After the treatment, complete improvement of the clinical picture with improvement of MDS is observed in: 22 $(88 \%)$, (MDS $=2.16)$ in therapy group with Bacterial colpitis, compared with $9(47 \%)$ in control group (Fisher's Exact test $\operatorname{mid} P=0,0065) ; 19(95 \%)$, (MDS=3.6) in therapy group with Trichomonas colpitis, compared with $8(50 \%)$ in control group (Fisher's Exact test mid $P=0.0046$ ) and $7(58 \%)$, (MDS $=3,7)$ in therapy group with Candida colpitis, compared with $3(33 \%)$ in control group (Fisher's Exact test mid $P=0.39$ ). Repeated PAP three months after treatment revealed "satisfactory but limited with inflammation" cytology in $11(19 \%)$ of the patients in therapy group.

Conclusion: Therapy with Polycresulen vaginal suppositories presents statistically significant improvement of the clinical picture and symptoms of recidivant bacterial and Trichomonas colpitis and improvement of recidivant Candida colpitis which is not statistically significant. The therapy significantly decreases the percent of "satisfactory but limited with inflammation" PAP findings in the patients with recidivant colpitis, that decreases the risk of false-negative PAP in this group of patients.

The Role of Diflucan in the Treatment of Mycoplasma Infection in Young Girls

S. Bagdâny, R. Mannah

Weiss Manfred Postgraduate Hospital, Department of Obstetrics and Gynecology, Budapest, Hungary

The treatment of vaginal mycosis is a great problem in the end of 20 th century. We can say, and it is a fact, that $50 \%$ of the young girls have different symptoms, such as vaginal flora or itchiness.

We have studied young girls in our region. There were 87 girls between 17-22 years. We observed candidiasis in $89 \%$ trichomoniasis in $2 \%$ and other infections in $9 \%$.
The treatment was Diflucan for 76 girls, $150 \mathrm{mg}$ given once and the bacterial investigation was made after a week of the treatment.

HPV 16 and 18 Infection in Patients With Cervical Intraepithelial Neoplasia and in Women Without Cervical Pathology

V. Kova, I. Takac, D. Zukanovic, T. Kodric, B. Gorisek

Gynecology and Perinatology Clinic, Maribor Teaching Hospital, Maribor, Slovenia

Background: During the past few years the number of studies dealing with human papillomaviruses (HPV) has increased rapidly as the discovery of new molecular methods made a more precise detection of virus DNA possible. But despite numerous studies, the role of HPV in the development of the neoplastic process remains unclear.

Methods: The aim of our study was to compare the incidence of HPV 16 and 18 infection in patients with cervical intraepithelial neoplasia (CIN) and in women without cervical pathology. In 246 patients treated for CIN with conization, prior to surgery cytologic material was collected for in situ hybridization (ISH) to prove HPV infection. The control group comprised 106 women without cervical pathology. In these women cytologic material for ISH was collected as well. The incidence of HPV 16 and 18 infection was also compared in individual CIN stages. The $\mathrm{z} 2$ test was used to calculate statistically significant differences.

Results: Among 246 patients with CIN, HPV (16 and/or 18) infection was present in $80(32.5 \%)$. HPV 16 infection was assessed in $38(15.4 \%)$, HPV 18 infection in $25(10.2 \%)$ patients. Both virus types were present in $17(6.9 \%)$ patients with CIN. In the control group, HPV infection was present in $35(33.0 \%)$ women. HPV 16 infection was assessed in 18 $(17.0 \%)$, HPV 18 infection in $14(13.2 \%)$ and infection with both HPV types in $3(2,8 \%)$ women.

Conclusion: There is no statistically significant difference between HPV (16 and/or 18) infection in patients with CIN and the control group. There is also no statistically significant difference between both groups as regards the incidence of infection with HPV 16 only and HPV 18 only. On the basis of our results obtained with the ISH method it is not possible to confirm the etiologic role of HPV 16 and 18 infection in CIN pathogenesis.

Preventive Effect of an Estradiol Releasing Vaginal Ring (Estring) on Recurrent Urinary Tract Infections (UTI) in Postmenopausal Women

Eriksen B.C.

Haugesund Gynecologic Clinic, Haugesund, Norway Introduction: An estradiol-releasing vaginal ring (Estring) containing $2 \mathrm{mg}$ of estradiol is available for the local treatment of postmenopausal urogenital symptoms. The primary objective of this study was to detect any difference in time to the first recurrence of UTI during treatment with Estring compared with no estrogen treatment in postmenopausal women. Material and Methods: This was a multicenter, randomised, open, parallel group study. A total of 108 postmenopausal women with recurrent symptomatic, bacteriologically confirmed UTI were randomly assigned, 53 to the Estring group and 55 to the control group. One ring was carried vaginally for 12 weeks. The duration of treatment was 36 weeks for 
the Estring group and either 36 weeks or until the first recurrence for the control group. The primary variable was analysed by survival analysis with the Kaplan-Meier method for estimating the survival density function. To compare the survival curves for the 2 treatment groups a log-rank test was performed for time until first recurrence.

Results: The cumulative proportion of women remaining free of UTI was significantly higher in the Estring group than in the control group $(P-0.008)$. After 36 weeks of study the cumulative likelihood of remaining free of disease was approximately $45 \%$ in the women with the vaginal ring compared with approximately $20 \%$ in the control group. Estring lowered vaginal $\mathrm{pH}$, and the time to first recurrence was effectively prolonged by Estring treatment. Vaginal and, to a lesser extent, urethral mucosal cells were significantly more mature in the Estring group. No unexpected adverse events were found.

Conclusion: Estring is useful to prolong the time to next recurrence among postmenopausal women with recurrent UTI and to decrease the number of recurrences per year. The silicone vaginal ring also has a clinically significant ability to alleviate other postmenopausal urogenital symptoms. Estring is safe and well tolerated.

\section{Serological Status of HBV, HCV, and HIV Among Preg- nant Women}

Yücel A,* Bozdayi G,** Imir T***

*Gazi University; Health Sciences Institute, Department of Microbiology, Section of Immunology, ${ }^{*}$ Gazi University, Health Sciences Institute, Dept. of Microbiotogy, ${ }^{* * *}$ Gazi University, Faculty of Medicine, Dept. of Microbiology, Section of Immunology

In this study, we aimed to determine the seroprevalence of hepatitis B virus (HBV), hepatitis C virus (HCV) and human immunodeficiency virus (HIV) infections among 644 pregnant women (mean age of $27.92 \pm 5.2$ years) evaluated in Gazi University Hospital Central Diagnostic Immunology Laboratory by means of tests ordered by their doctors in the Department of Obstetrics and Gynaecology during the last 18 months. Not all tests below were ordered for each woman by obstetricians. All tests were performed by means of a very sensitive, automatic paramagnetic chemiluminescent immunoassay system. Our results may be summarised as follows:

Table 1: HBV, HCV, and HIV seropositivity among 644 pregnant women

\begin{tabular}{lcc}
\hline Serological marker & Total $(\mathrm{n})$ & Positive \\
\hline HBsAg & 632 & $20(3.2 \%)$ \\
Anti-HBs & 246 & $93(37.8 \%)^{*}$ \\
Anti-HCV & 168 & 0 \\
Ant-HIV & 213 & $1(0.5 \%)^{* *}$ \\
\hline
\end{tabular}

*Anti-HBs titers equal or above $10 \mathrm{mIU} / \mathrm{ml}$ were accepted as positive for protection against HBV infection. Of these 93 anti-HBs $(+)$ women, mean antibody titer was $235.10 \pm$ $212.13 \mathrm{mIU} / \mathrm{ml}$ (minimum $12 \mathrm{mIU} / \mathrm{ml}$. maximum $650 \mathrm{mIU} /$ $\mathrm{ml})$.

** This patient was tested twice for anti-HIV antibody in our laboratory and both results were positive. According to legal regulations. patient was transferred to a governmental reference laboratory for confirmation by Western Blot; final result there was reported as "negative".
Table 2: HBV, HCV, and HIV serology of the 168 pregnant women

\begin{tabular}{lcc}
\hline Serology & Number & Percent \\
\hline $\begin{array}{l}\text { HBsAg(-), anti-HBs(+), } \\
\text { anti-HCV (-), anti-HIV(+) }\end{array}$ & 1 & $0.6 \%$ \\
$\begin{array}{l}\text { HBsAg(+), anti-HBs(-), } \\
\text { anti-HCV (-), anti-HIV(+) }\end{array}$ & 5 & $3.0 \%$ \\
$\begin{array}{l}\text { HBsAg(-), anti-HBs }(+), \\
\text { anti-HCV (-), anti-HIV(-) }\end{array}$ & 56 & $33.3 \%$ \\
$\begin{array}{l}\text { HbsAg(-), anti-HBs(-). } \\
\text { anti-HCV (-), anti-HIV }(+)\end{array}$ & 106 & $63.1 \%$ \\
TOTAL & 168 & $100 \%$ \\
\hline
\end{tabular}

It is seen that in routine followup of pregnant women usually only HBsAg among above markers was ordered. Thinking of the dramatic outcome of any possible HCV or HIV infection in pregnancy, we suggest that anti-HIV antibody and anti-HCV antibody tests should also be included to prevent any risk to the fetus; and that it would be better (from both an immunological and economical point of view) to test at first anti-HBc total antibody (both IgM and IgG against core antigen of $\mathrm{HBV}$ ), where it is available, rather than $\mathrm{HBsAg}$ alone or HBsAg and anti-HBs together to show any sign of exposure to HBV. If anti-HBc total is found to be negative; there will be no need for further tests; however, if it is found to be positive, then it would be better to investigate the whole serologic panel for HBV.

Scientific Medical Diagnostic Centre's “Astar" (LVIV, Ukraine) Four-Years Work Report

Sluzhynska O.M.

Scientific Medical Diagnostc Centre "Astar", Lviv, Ukraine Scientific Medical Diagnostic Centre "ASTAR" works as the STD centre dealing with diagnostics and treatment of the STIs. It is the first and the only centre in the area of the Western Ukraine working in this field. The Centre's staff consists of 14 persons. Since the beginning of work the centre has had more than 4000 visitors.

During the period of work the Centre has had an increasing number of patients. Chlamydia trachomatis has been diagnosed in $65 \%$ of them. Trichomonas vaginalis in $-41 \%$. These two STIs are the most widespread in the Western Ukraine. Mixed STIs are more often (89\%) than mominfections. 65\% of the Centre's visitors are women. Many of the women are victims of trafficking in women and victims of rape.

The centre is looking for the possibility of cooperation and developing joint activities with foreign STI centres.

\footnotetext{
HBV Prevalence in Patients With Gynecologic Malignancy

Cüce $\mathrm{M},{ }^{*}$ Hanhan $\mathrm{M},{ }^{* *}$ Gürsoy G.**

Infectious Disease Specialist, ${ }^{* *}$ Gynecology and Obstetrics Specialist

SSK Ege Gynecology and Obstetrics Disease Hospital, Izmir, Turkey

The incidence of Hepatitis :B virus (HBV) is reported in normal population and various risk groups in Turkey. In our study
} 
we searched HBV incidence in the patients with gynecologic malignancy, who were relatively less studied.

Sera samples were obtained between January 1999 and September 1998 in SSK Ege Obstetrics and Gynecology Hospital. $\mathrm{HBs} \mathrm{Ag}$ and $\mathrm{HBs} \mathrm{Ab}$ were detected by Vidas ELFA technique. $\mathrm{HBs} \mathrm{Ag}$ positivity was found in $21(6.6 \%)$ and $\mathrm{HBs} \mathrm{Ab}$ in 115 $(36.4 \%)$ of 316 patients. $180(57 \%)$ patients were found seronegative for $\mathrm{HBV}$.

The incidence was found to be the same as normal population.

Histopathologic Changes of the Fallopian Tube in Women Using an Intrauterine Contraceptive Device (IUCD) Inan $\mathrm{S}$, * Vatansever HS, * Yagceken $\mathrm{S},{ }^{* *}$ Benli $\mathrm{Z}$, ** Ertopcu $\mathrm{K}$,** Ozelmas I**

*Dept. of Histology \& Embryology, Faculty of Medicine, Celal Bayar University, Manisa

**Dept. of Family Planning, Aegean Social Insurance Inst., Obstetric \& Gynecology Hospital, Izmir, Turkey

The use of IUCD is becoming more widespread. It has been a reliable and reversible contraceptive alternative for more than 30 years. By preventing the spermatozoa from reaching the fallopian tube, ovum transport and fertilization; it is a means of protection against pregnancy.

The aim of this study was to investigate and evaluate the histopathological changes in the fallopian tube in women using the IUCD (multiload Cu375 \& T Cu380A) for a long period of time. Isthmic portions of the fallopian tubes were obtained from twenty healthy women by tubal ligation, using the Pomeroy technique. Specimens were fixed in $10 \%$ formalin and followed in a routine manner. Sections were stained with Hematoxylin-Eosin (H.E.) and Toluidin blue; then evaluated under the light microscope.

Using a light microscope, a pronounced reduction of ciliated cells in the epithelium of fallopian tube, increased number of mast cells, and inflammatory cell infiltration were found in the lamina propia in IUCD users as compared to nonusers, in the same menstrual period. Mast cells are known to contain potent biological mediators and these may be responsible for local inflammatory reactions. These findings may be a factor in the pathogenesis of pelvic inflammatory disease and ectopic pregnancies that occur in IUCD.

\section{Epidemiological Features and Microbial Etiology in Turk- ish Women With Cervicitis}

Tuzcular Vural EZ, Terzioilu S, Kaynar Taiçioilu J, Yazicioilu E

Haydarpaia Numune State Hospital (HNSH) Istanbul, Turkey

Objective: Cervicitis is one of the most frequent genital infections among women of the reproductive age. The aim of this study was to evaluate microbial etiology and epidemiological features in Turkish women with cervicitis.

Materials and Methods: 40 randomly selected women presenting with cervicitis to the Family Planning Clinic of HNSH were included in this study. Patients under 18 and over 50 years of age, women who had received antimicrobial drugs within the prior month, and menstruating women were excluded. Cervical smears, vaginal and endocervical specimens, and gram-stains were obtained from all patients.

Results: The average age of the patients was 29.2 years (range
20-40). The average number of pregnancies was 4.1 (range $1-11$ ) and the average number of living children was 2.7 (range 1-6). The most common complaints were leucorrhoea $(82.5 \%)$, lower abdominal pain (42.5\%), lombalgia (17.5\%), postcoital bleeding (7.5\%), dyspareunia $(5 \%)$, urinary symptoms $(2.5 \%)$ and pruritus $(2.5 \%) .10 \%$ of the patients had no complaint. 39 of the smear results were normal (Class I), with one Class II result. $42.5 \%$ of the patients had an IUD, $27.5 \%$ practiced coitus interruptus, $15 \%$ used condoms, $5 \%$ oral contraceptives, and $7.5 \%$ used other methods. The microbial flora revealed Chlamydia trachomatis (7.5\%), Neisseria gonorrhoeae (7.5\%), Ureaplasma urealyticum (35\%), Mycoplasma hominis (17.5\%), bacterial vaginosis $(25 \%)$, Proteus mirabilis $(5 \%)$, Trichomonas vaginalis $(2.5 \%)$ and streptococci $(12.5 \%)$ as etiological agents. All patients were married and had apparently just one sexual partner.

Conclusion: Cervicitis is of importance especially because of its association with serious upper genital tract infections. Considering that all women apparently had only one sexual partner, the overall rate of microbial etiology capable of resulting in PID was higher than we expected.

\section{Hepatitis B Screening Results in Pregnant Women} Kesim M., Kaya I., Yalçin A., Erdemir M., Öztürk H. Iiili Etfal Hospital 3. Obstetrics and Gynecology Clinic, Istanbul,Turkey.

Objective: We aimed to investigate hepatitis B porters among pregnant women.

Materials and Methods: Among 294 pregnant women who have attended to Iiili Etfal Hospital 3rd Obstetrics and Gynecology outpatient clinic, HBsAg, AntiHBs, and in HBsAg positive pregnant women, $\mathrm{HBeAg}$ were investigated by macro Elisa system.

Results: Among 294 pregnant women $\mathrm{HBsAg}$ was positive in 22 women $(7.48 \%)$, antiHBs was positive in 44 women $(14.96 \%)$. In 22 pregnant women in whom $\mathrm{HBsAg}$ were positive, $\mathrm{HBcAg}$ was also found positive.

Conclusion: Since hepatitis B virus can cause serious medical problems, we aimed to point out the importance of hepatitis B screening for the health of the mother and newborn and so that it should not be forgotten during routine laboratory testing of pregnant women.

\section{Pelvic Inflammatory Disease}

Lazdane G., Viberga I.

Medical Academy of Latvia, Riga, Latvia

Introduction: Topicality of the problem of pelvic inflammatory disease (PID) is due to its high incidence in the countries of Eastern Europe. The combination of induced abortion with widely spread sexually transmitted diseases (STD) directly influences the reproductive health of the population and causes infertility. The goal of the study is to evaluate the factors influencing the incidence of PID, family planning practice in Latvia and the relation of different methods of contraception with PID.

Materials and Methods: The results of the survey on reproductive health and sexual behaviour of the population of Latvia ( 2990 women were interviewed), the study of the role of intrauterine contraception (IUD) in the epidemiology of PID (127 hospitalized PID patients and 100 women in the control group), the study of the role of induced abortion in 
the epidemiology of PID (the analysis of the medical history of 126 hospitalized PID patients with tuboovarian abscesses) are included.

Results: $26.6 \%$ of women in Latvia have started their sexual activity prior to the age of $17,24.5 \%$ of sexually active women in the age group of 15-19 have had 4 or more sexual partners. The number of IUD users among the patients hospitalized due to PID was two times higher than among the control group $(P<0.005) .40 .48 \%$ of patients with tuboovarian abscesses stated that the first episode of PID was related to an induced abortion.

Conclusion: The early sexual activity of young people and lack of knowledge about safe sex increases the incidence of PID and is to be considered a serious health care hazard and an educational problem in Latvia. IUD should be recommended after the risk of STD has been evaluated, the screening of urogenital chlamydiosis is recommended. Patients under the age of 25 who come for an induced abortion are to be screened for urogenital chlamydiosis before the abortion or preventive doxocycline treatment is to be prescribed.

\section{Serological Status of TORCHE Infections Among Preg- nant Women}

Yücel $\mathrm{A},{ }^{*}$ Bozdayi G,** Imir T.***

*Gazi Universitv. Health Sciences Institute, Department of Microbiology, Section of Immunology; Ankara **G.U. Health Sciences Institute. Dept. of Microbiology, Ankara

***G.U. Faculty of Medicine, Dept. of Microbiology, Section of Immunology, Ankara, Turkey

In this study, we aimed to determine the seroprevalence of TORCHE infections among 740 pregnant women (mean age of $27.1 \pm 8.3$ years) evaluated in Gazi University Hospital Central Diagnostic Immunology Laboratory by means of tests demanded bv their doctors in the Department of Obstetrics and Gynaecology during last 18 months. Not all tests below were demanded for each woman by their obstetricians. IgM and IgG antibodies against toxoplasma and rubella were measured by a new sensitive. automatic chemiluminescent paramagnetic immunoassay system. All other tests were performed by commercially available manual ELISA kits. Our results may be summarized as follows:

Table 1: TORCHE seropositivity among pregnant women

\begin{tabular}{lcc}
\hline Specific antibodies & Total $(\mathrm{n})$ & Positive \\
\hline Toxoplasma IgM & 394 & $11(2.8 \%)$ \\
Toxoplasma IgG & 690 & $214(31 \%)$ \\
Rubella IgM & 271 & $5(1.8 \%)$ \\
Rubella IgG & 670 & $627(93.6 \%)$ \\
HSV-I IgM & 80 & $6(7.5 \%)$ \\
HSV-II IgM & 79 & $6(7.6 \%)$ \\
EBV IgM & 47 & $1(2.1 \%)$ \\
CMV IgM & 109 & $0(0 \%)$ \\
CMV IgG & 148 & $137(92.6 \%)$ \\
\hline
\end{tabular}

Table 2: IgG and IgM results for rubella, toxoplasma and CMV

\begin{tabular}{llllll}
\hline $\begin{array}{l}\text { TORCHE } \\
\text { Agent }\end{array}$ & $\begin{array}{l}\text { TOTAL } \\
(\mathbf{n})\end{array}$ & $\begin{array}{l}\operatorname{IgM}(-) / \\
\operatorname{IgG}(-)\end{array}$ & $\begin{array}{l}\operatorname{IgM}(+) / \\
\operatorname{IgG}(-)\end{array}$ & $\begin{array}{l}\operatorname{IgM}(-) / \\
\operatorname{IgG}(+)\end{array}$ & $\begin{array}{l}\operatorname{IgM}(+) / \\
\operatorname{IgG}(+)\end{array}$ \\
\hline Toxoplasma & 361 & $252(69.8 \%)$ & $1(0.3 \%)$ & $96(26.6 \%)$ & $12(3.3 \%)$ \\
Rubella & 246 & $21(8.5 \%)$ & - & $223(90.7 \%)$ & $2(0.8 \%)$ \\
CMV & 84 & $4(4.8 \%)$ & - & $80(95.2 \%)$ & - \\
\hline
\end{tabular}

As seen in Table 2. IgM and IgG seropositiviy may occur at the same time during course of infections. Therefore, we suggest that all pregnant women should be evaluated for TORCHE IgM's as well as TORCHE IgG's in the first visit to their obstetricians (especially for toxoplasma and rubella) otherwise it is possible to not notice an acute TORCHE infection in pregnancy if only TORCHE IgG's are tested because the patient may be asymptomatic although the complications on fetus may be revealed later. However, IgM seropositivity does not always mean an acute toxoplasma infection that started during pregnancy. In this case, the use of toxoplasma IgG avidity test has been suggested recently for differentiation of an acute infection during pregnancy.

\section{Clindamycin Cream Versus Oral Metronidazole in the} Treatment of Bacterial Vaginosis

Sezen D, Tuzcular Vural EZ, Yazicioilu E

Haydarpaia Numune State Hospital, Gynecology and Obstetrics Department, Istanbul, Turkey

Objective: Bacterial vaginosis (BV) is characterized by the replacement of the vaginal flora which is normally dominated by lactobacillus species by Gardnerella vaginalis and anaerobic bacteria. BV is the most common vaginal complaint among women of reproductive age and is associated with serious complications. The objective of this study was to compare the efficacy of $2 \%$ clindamycin vaginal cream versus oral metronidazole in the treatment of BV.

Materials and Methods: 60 patients diagnosed as BV with clinical criteria supported by gram-stain as described by Amsel and culture were divided randomly into two equal groups. One group received oral metronidazole $500 \mathrm{mg}$ twice a day for 7 days and the second group $2 \%$ clindamycin vaginal cream $5 \mathrm{gm}$ once a day for 7 days. Cure rates and side effects were evaluated by a return visit on the 15 th day.

Results: There was no significant difference $(P>0.05)$ between the cure rate of oral metronidazole and clindamycin vaginal cream $(86.7 \%$ and $83.3 \%$ respectively). Side effects were nausea $(13.3 \%)$, metallic taste $(6.7 \%)$, candidal vaginitis $(3.3 \%)$ for oral metronidazole and vaginal irritation $(30 \%)$ and candidal vaginitis $(13.3 \%)$ for clindamycin cream.

Conclusion: Clindamycin vaginal cream $(2 \%)$ is a safe and effective alternative to oral metronidazole for treatment of BV.

\section{Prevalence of Mobiluncus Species in Turkish Patients With Gynecologic and Obstetric Infections}

Bahar H., Mamal Torun M.

Istanbul University,Medical Faculty of Cerrahpaia, Department of Microbiology and Clinical Microbiology, IstanbuI, Turkey

Mobiluncus species are especially found in the vaginal flora of women with bacterial vaginosis (BV) and their presence in the vaginal samples were accepted as a good indicator of BV.These microorganisms may occasionally ascend to the upper genital tract of females and they have been isolated from cases of endometritis and salpingitis.

The aim of this study was to determine the prevalence of Mobiluncus sp. in 364 specimens (102 specimens from vulvovaginal abscesses, 42 specimens from cases of endometritis, 26 specimens from cases of salpingitis and 194 specimens of vaginal smears which microscopic exams revealed flora compatible with BV) investigated in the period between 
May 1996 and May 1999. Mobiluncus strains were isolated in Schaedler agar and in RLK agar at anaerobic atmosphere and were identified using their biochemical characteristics. The results were confirmed with API $20 \mathrm{~A}$ and SCEPTOR anaerobic ID panels.

The prevalence of Mobiluncus sp. in pus of 102 vulvovaginal abscesses was found to be $2 \%$ ( 2 strains of M.curtisii), the prevalence in 42 specimens of endometrial cultures was found to be $4.7 \%$ (2 strains of M.curtisii) and in 26 specimens of pus from tubes of cases with salpingitis was found $3.8 \%$ (1 strain of M.curtisii).The prevalence in 194 vaginal samples was found $77.2 \%$ using Gram staining and after their isolations this prevalence was $49 \%$ ( 70 strains were M.curtisii and 25 strains were $M$. mulieris.)

As a result of this research we determine that Mobiluncus species which are frequently found in the vagina of women with $\mathrm{BV}$ can be one of the important agents to cause vulvovaginal abscesses, endometritis, and salpingitis.

\section{Is Vaginal Douching a Risk Factor For Bacterial Vaginosis?}

Tilli, M, ${ }^{*}$ Farinati,A, ${ }^{*}$ Saine, $M,{ }^{* *}$ Mormandi, JO, Petracini, $\mathrm{N}^{*}$ and Levin, $\mathrm{A}^{*}$

*Hospital Eva Perön and **Hospital Fernandez. Buenos Aires, Argentina

Aim: To investigate the relationship between vaginal douching (VD) and bacterial vaginosis (BV) and to determine if it is a risk factor to promote this infection.

Study Design: This is a control-case study. Four hundred one (401) women with vaginal discharge were studied at the Control Infectious Diseases Section of HIGA Eva Perön, from Jan 1997 to Dec 1998. We selected 103 of them with BV or Normal Flora (NF) and they were separated in two prospective groups: BV (group 1, n=40) and NF (group 2, n=63). We excluded the pregnant women, patients with local or systemic antimicrobial therapy, other local therapy and other genital tract infection. The VD was studied as a primary variable and contraceptive methods, number of partners and sexual behaviours as secondary variables. Statistical analysis was performed with EPI INFO version 6.2, odds ratio (OR) and the $95 \%$ confidence intervals $(\mathrm{CI})$ for the difference between proportions as well as p values for probability test were calculated. $P<0.05$ was considered significant.

Results: No statistical differences were found between both groups in age, contraceptive methods and sexual behaviour, the only exception was the primary variable studied. The VD were present in $29 / 40(72.5 \%)$ and absent in 11/40 (27.5\%) of women with BV and in 20/63 (31.7\%) and 43/63 (68.3\%) respectively, of NF group $(P<0.0001$, OR : 5.67 and $95 \%$ CI : 2.19-15.04).

Conclusion: The multifactorial pathogenesis of $\mathrm{BV}$ is a problem in an attempt to control this genital tract infection. Because the VD was significantly associated with BV women, it would be considered a risk factor for this infection, probably due to normal flora alterations and clearance of Lactobacillus spp. The other factors considered had no influence on this group of women.

STD Infections in Women of Central Asia: Preliminary Results of WHO Surveys

Stray-Pedersen B.

Dept of Obstetrics and Gynecology, National Hospital, University of Oslo, Oslo, Norway
In the last decade countries in the former Soviet Union have experienced a dramatic increase in the incidence of STDs. Population based surveys have been performed, both in Tajikistan and Turkmenistan, initiated by the governments, WHO and UNFPA.

In Tajikistan nearly 400 women from the same village in the rural region of Khaton were examined, and in nearly $40 \%$ of the cases different STDs were discovered. These were Trichomoniasis $25.3 \%$, Chlamydia trachomatis $14.9 \%$, gonorrhoea $0.2 \%$, syphilis $5.6 \%$.

In Turkmenistan 600 women have been studied, including pregnant women, women are applying for abortion, teenagers, and women with chronic pelvic pain. The preliminary results were alarming, showing a very high rate of STDs including syphilis, gonorrhoea, trichomoniasis, chlamydia, and hepatitis B and C in each group. In both countries and the other states of Central Asia there is a urgent need to increase the population's awareness of STDs.

Knowledge, education and perception of STDs are important. Up to date laboratory facilities and avaibility of medication are cornerstones in combating the arising epidemic.

The Value of Transvaginal Color Doppler in the Assessment of Pelvic Inflammatory Disease

A. Kurjak, S. Kupesic

Department of Obstetrics and Gynecology, Medical School University of Zagreb, Sveti Duh Hospital, Zagreb, Croatia

Objective: To compare transvaginal color Doppler, laparoscopic and clinical findings in 102 women with proven pelvic inflammatory disease (PID).

Materials and Methods: All the patients were examined by transvaginal Color and pulsed Doppler ultrasound (Aloka SSD 2000).

Results: In patients with acute PID $(\mathrm{N}=72)$, increased endometrial thickness was detected. Increased endometrial vascularity $(\mathrm{RI}=0.50 \pm 0.05)$ was the only uterine abnormality demonstrated in $49(68 \%)$ patients. Color signals were obtained from septal parts or external margins in $56(75.7 \%)$ patients presenting with complex adnexal masses. Tubal arteries were identified in $12(54.6 \%)$ patients with tubular anechoic adnexal structures. In the early phase of PID ovarian parenchymal blood flow was characterized with low-tomoderate resistance to blood flow $(\mathrm{RI}=0.53 \pm 0.09)$. In the chronic stage the $\mathrm{RI}$ was increased $(\mathrm{RI}=0.71 \pm 0.09, P<0.05)$. Conclusions: Transvaginal color and pulsed Doppler is a useful additional tool in diagnosis and treatment monitoring of patients with PID. This method may be useful in differentiating hydrosalpynx from dilated veins in patients suffering from pelvic congestion syndrome, or pyosalpynx from hematosalpynx in patient with ectopic pregnancy.

\section{Prevention of Congenital Toxoplasmosis}

Stray-Pedersen B

Dept of Obstetrics and Gynecology, National hospital, University of Oslo, Oslo, Norway

Toxoplasma infections are widespread and occur all over Europe. Exposure to infections varies regionally. From 30 to 90 percent of pregnant woman are nonimmune and susceptible to primary infection, $1-5$ per 1000 women acquire infection during pregnancy, while the overall fetal transmis- 
sion rate is 40 percent. Congenital toxoplasmosis is a preventable disease. In 1989 WHO-Europe encouraged countries to consider antenatal screening programs.

In 1992 a European Toxoplasma Research Network was established. Several groups have been working together and new data are now available.

Primary prevention includes application of certain dietetic and hygenic measures which can reduce the infection rate. Today's advice given to seronegative pregnant women is to avoid ingestion of contaminated or infected food (undercooked meat from lamb, beef and game, unwashed fruits and vegetables), and to avoid oocyts from cat faeces (use gloves and handwashing after contact with sand soil, and cat litter boxes). Susceptible women living in "low risk" areas should avoid traveling to "high risk" areas outside Europe and North America during pregnancy.

Secondary prevention comprises serologic testing at regular intervals during pregnancy. This allows for identification of women acquiring the infection during gestation. In these risk cases prenatal diagnosis (amniocentesis) should be performed to identify infected fetus. A new European multicentre study indicates that therapy has a great impact on the outcome of congenital infection with a significant reduction in severe sequelae at one year of age. There are also new guidelines in treatment of newborns.

\section{Syphilis Screening in Pregnancy - Is It Still Important?} Stray-Pedersen B.

Dept of Obstetrics and Gynecology, National Hospital, University of Oslo, Oslo, Norway.

Congenital syphilis can be largely prevented by identification and treatment of the infected women during pregnancy. Since primary syphilis is symptomatic, identification requires serologic screening. In most European countries serologic testing of all pregnant women has been recommended for 40 years. During the years 1980-1990 the incidence of syphilis in most countries of Western Europe has dropped below 2 per 100,000 population.

In the Scandinavian countries the incidence is now below 1 per 100,000 . The question has thus been raised, if the obligatory prenatal screening program ought to be discontinued.

In Norway during the last 10 years, from 1989-1998, approximately 650,000 pregnancies have been screened and only 59 cases of syphilis have been identified. In 9 cases serology and history were suggestive of early infectious syphilis while in the remainder latent infections ( $>1$ year) were identified. Approximately half of the cases were immigrants. All women were treated and delivered live born infants without stigmata of congenital syphilis. Taking into consideration a vertical transmission rate of $90 \%$ in early syphilis and $10 \%$ in late syphilis, 13 children were expected to be infected without a screening program. In addition, in this period 11 cases of congenital syphilis were detected, all were adopted or immigrant children from Asia or South Africa.

Even in a country with a very low sero prevalence the antenatal screening program (using TPPA or TPHA which detect latent infections), is found to be cost effective. Thus, it is decided that routine screening should be continued. The antenatal screening is today the only syphilis surveillance in our country and is of importance since our neighbours in Russia and the Baltics at present experience a syphilis epidemic with a 100 fold increase. In these countries pregnant women should be tested twice, at the first antenatal visit and repeated at the end of pregnancy.

\section{Chronic Toxoplasmosis and Its Treatment During Preg- nancy}

Prof. Dr. Sevim Dinçer

CENGIZ,Ankara University Faculty of Medicine, Department of Obstetrics and Gynecology

Epidemiological evidence suggests that $25-50 \%$ of world's population have been infected by Toxoplasma gondii. The seropositivity can be as high as $90 \%$ especially in countries consuming raw meat such as Turkey. The cyst form is responsible for persistence of latent infection in multiple organs as brain, heart muscle, and skeletal muscle where they persist throughout the life of the host. In immunocompromisod carriers such as HIV patients, malignancy or in the absence of maternal immune response in pregnancy, reactivation of the infection may occur and parasitenva is seen. This leads to disruption of llssuc cysts and release of trophozites into the circulation. Fetal infection can be observed during the primary infection in pregnancy. The passage is usually seen in the last trimester but the damage caused by the infection is smaller. Fetal infection usually leads to fetal demise in the first trimester or may cause multiple organ defects. Reactivation of toxoplasmosis usually presents with habitual abortion, preterm labor and fetal demise. There is evidence that some chronically infected mothers have foci of Toxoplasma gondii in the uterus. In patients with above mentioned pathologies the possibility of a reactivation during pregnancy must be kept in mind. We have been studying chronic toxoplasmosis during pregnancy since 1975. After ruling out all reasons for the above mentioned pathologies, the treatment of patients with toxoplasmosis remarkably increased the rate of live births. In the beginning of this study, Sabin-Feldman dye test and indirect fluorescence antibody tests were utilized for the diagnosis of toxoplasmosis. Recently we have been using polymerase chain reaction (PCR) to detect the active infection. Due to reactivation of the CNS, toxoplasmosis in AIDS cases of chronic disease gained greater interest which led to discussion of new diagnostic tools such as PCR and new treatment modalities. Treatment is only indicated when a clear therapeutic benefit can be demonstrated. We have been using pyrimethamine since 1975 for the treatment of toxoplasmosis during pregnancy. Although it has teratogenic potential we have not observed any teratogenic effects of the therapy in our series. The success of the therapy depends on the timing of the treatment and the treatment must be initiated as early as weeks 7 or 8 of pregnancy.

Due to the known side effects of high dose pyrimethamine therapy we developed our "attack" treatment. The schema of treatment (Dinçer formula) is as follows $3 \times 25 \mathrm{mg}$ for first 3 days, $2 \times 25 \mathrm{mg}$ for the following three days and $1 \times 25 \mathrm{mg}$ for 1 month. This drug is combined with folinic acid since pyrimethamine is a folic acid antagonist. The treatment was monitored with IgG titers at week 20 of pregnancy. If the titers were high then spiramycine was administrated as a new line of therapy.

Feminine Hygiene Practice Among Health Care Personel in Turkey

Saracoglu OF, Yilmaz N, Eiilmez P, Akçul H

Numune Resarch and Training Hospital, Dept of Obstetrics and Gynecology, Ankara, Turkey 
Aim: Research in the area of feminine hygiene practice of Turkish women is limited. In the present study information related to feminine hygiene practices among nurses was searched.

Method: A descriptive research design was used to examine feminine hygiene practices among 250 nurses who were 20 years of age or older, working at the Ankara Numune Research and Training Hospital. A questionnaire containing 28 questions about feminine hygiene was developed after a literature review.

Results : All the participants were Muslim. 71\% were married, $18 \%$ divorced and $11 \%$ single. $26 \%$ reported that they took a shower everyday, $62 \%$ took a bath every $2-3$ days, whereas $1 \%$ used a tub for bathing and $98 \%$ used a handheld shower. $22 \%$ of the participants reported never douching vaginally whereas $29 \%$ douched sometitimes and only $49 \%$ always used an internal douche. $12.5 \%$ of the participants used panty liners during intermenstrual period, whereas most never used feminine hygiene spray (3\%), deodorant vaginal tablets or suppositories. Wet wipes were used by $4 \%$ of the nurses and the practice of wiping from front to back was always done by $90 \%$ of them. Hands were always washed after urination/defecation by $92 \%$ of them. During menstruation $62 \%$ of the nurses used sanitary napkins and less than $1 \%$ of them used tampons. $50 \%$ of the responders washed their hands before and after changing their pads or tampons. When they used sanitary napkins $57 \%$ of them changed them at least every 2-4 hours, if they used tampons $\%$ of them changed it at every 2 hour interval.

Conclusion : Results of the study shows that even if the participants were health personnel it is important to teach women some correct feminine hygiene practices.

The Comparison of Piperacillin Use for Prophylaxis of Post Cesarean Section Infection Before and After Clamping Umblical Cord

Gül A, Zeteroilu I, Sürücü R

Yüzüncü Yil University, Faculty of Medicine, Dept of Obstetrics and Gynecology, Van, Turkey

Objective: We aimed to research efficacy of piperacillin use for prophylaxis of cesarean section infection before making incision and after clamping umblical cord.

Material and Methods: A prospective randomized trial enrolled 57 pregnant women undergoing abdominal cesarean section due to any indication in our clinic during the period between February to September 1999. The sociodemographic status, complaints, and preoperative and postoperative physical findings, together with laboratory findings, indication for cesarean section, and duration of hospitalization of all patients were recorded onto previously prepared forms. Also the weight, sex, Apgar score and physical examination findings of all neonates were recorded and they were followed by us together with the neonatal care unit. 30 pregnant women were randomly assigned to receive 1 gr piperacillin intravenously before making incision and to 27 other pregnant women, piperacillin was administered intravenously immediately after clamping umblical cord following delivery of fetus. Totally 3 doses of piperacillin were administered to women with 12 hours interval.

Results: The findings of maternal and neonatal demographic status were similar in two groups. There was no significant difference between two groups by means of predisposing risk factors causing infection in postcesarean section period. The maternal diagnosis of maternal infection included occurrence of fever, high white blood cell count, endometritis, septic thrombophlebitis, pneumonia, urinary tract infection and opening or infection of the incision line. In first group that piperacillin was administered to before making incision, as a postoperative complication only induration was developed in $2(7.1 \%)$ patients. In other group that receiving piperacillin immediately after clamping umblical cord, induration. opening of incision line, and endometritis in cases of $1(3.8 \%)$, $1(3.8 \%)$ and $1(3.8 \%)$, respectively, were seen as postoperative complications. So, there was no significant difference between two groups by means of postoperative complication rate. In addition to that no neonatal infection developed in either group.

Conclusion: Abdominal cesarean section is the most commonly applied operation in obstetric surgery. Widespreadly, for prophylaxis after ceserean section, antibiotic is given immediately after clamping umbilical cord. Although no significant difference was detected according to postoperative complications between two groups in this study, we believe that antibiotic for prophylaxis must be administered at preoperative period to reach effective blood dose levels during operation by obeying prophylaxis rules. In our opinion, better results may be obtained in maternal and neonatal morbidity.

\section{Transvaginal Sonography in Detection of Pelvic Inflam-} matory Disease

A. Kurjak, S. Kupesic, T. Zodan, I. Bekavac

Department of Obstetrics and Gynecology, Medical School University of Zagreb, Sveti Duh Hospital, Zagreb, Croatia Objective: To assess the value of B-mode transvaginal sonography in the evaluation of the patients with laparoscopically proven pelvic inflammatory disease.

Materials and Methods: Seventy-two patients with acute symptoms of pelvic inflammation, 11 patients with chronic pelvic pain and 19 patients with infertility suspected of tubal etiology were admitted to our department. Endometrial thickness and morphology were evaluated together with ovarian measurements and morphological appearance. The equipment used in the study was Aloka SSD 2000 with transvaginal transducer of $5 \mathrm{MHz}$.

Results: Endometrial morphology suggestive of endometritis was demonstrated in 72 patients $(70.6 \%)$. Free fluid in the cul-de-sac was demonstrated in $39(38.2 \%)$ patients. Ovarian enlargement as the only finding was demonstrated in 6 $(5.9 \%)$ patients, while $22(21.6 \%)$ presented tubular adnexal structures. In $74(72.5 \%)$ patients transvaginal sonography detected complex adnexal mass.

Conclusions: Transvaginal sonography is a useful tool in detection of patients with pelvic inflammatory disease. In acute stages one can detect both uterine and adnexal findings (increased endometrial thickness and echogenicity, ovarian enlargement and/or complex adnexal mass). However, in chronic stages the most common finding is tubular adnexal structure presenting dilated tube without changes in uterine and ovarian morphology.

Prevalance of Bacterial Vaginosis in Patients With Various Gynecological Complaints

Petek Deniz Uçkan, Fadil Kara, Salim Erkaya, Serdar Erçakmak 
*Zübeyde Hanim Doiumevi, Microbiology and Clinic Microbiology Laboratory.

${ }^{* *}$ Zübeyde Hanim Doiumevi, Obstetrics and Gynecology Service

Bacterial vaginosis is the most prevalent vaginal infection in reproductive women. A group of microorganisms are present concurrently in high concentrations in the vagina of women with bacterial vaginosis. The major members of the group are Gardnerella vaginalis, Bacteroides species, Pestostreptococcus species, Mobiluncus species, Prevotella species and genital Mycoplasmas. The objective of this clinical trial was investigation of bacterial vaginosis in 313 patients presenting with various gynecological complaints. In 74 (24\%) patients clue cells and whiff test were positive and $G$ vaginalis was isolated.All these patients had profuse, homogenous and malodorous discharge and took the diagnosis of bacterial vaginosis. Of these 74 patients with bacterial vaginosis, 4 had concomitant Trichomonas vaginalis and 10 had Candida spp. infection. Among women with bacterial vaginosis there is a significantly increased risk of salpingitis, particularly following abortion, and of vaginal cuff and wound, or infection after hysterectomy.

Hence, bacterial vaginosis is a curable disease; diagnosis and treatment are essential to prevent complications.

\section{Pelvic Tuberculosis}

Saracoglu OF, Tanzer F, Türkkani B

Ankara Numune Research and Training Hospital, Dept Ob\&Gyn, Ankara, Turkey

Aim: Even if it is not common, pelvic tuberculosis is still a health problem in developing countries. In the present study our aim was to search the clinical and laboratory findings of these patients.

Materials and Method: Retrospectively the files of the patients in which pelvic tuberculosis was diagnosed (n: 78 ) during the years 1979-1999 were analyzed.

Results: Twenty-nine percent of the patients were over 40 years of age. The most common complaints were infertility $(\mathrm{n}: 34,43.5 \%)$, abdominal or pelvic pain $(\mathrm{n}: 25,32 \%)$ and abnormal uterine bleeding (n: $12,15.3 \%$ ). Only 2 patients had past or family history of pulmonary tuberculosis. The physical examination was normal in $31.6 \%$ of the patients. The chest X-ray was normal in $81 \%$ of the patients. Blocked tubes were present in 32 of 34 patients who had hysterosalpingograms. Even if the diagnosis can be made from a histopathologic examination, hysterosalpingography is also a very useful aid in establishing a diagnosis. The most common site of infection was the tubes. Reconstructive surgery was performed in nine patients. Only one pregnancy was found in the present study without any medical or surgical treatment of pelvic tuberculosis. In one patient with habitual abortion endometrial tuberculosis was also found as a cause.

Conclusion: Pelvic tuberculosis is a serious but rare disease in our population. However it must be in mind in infertile population. Although the diagnosis is made by endometrial biopsies in all the patients with infertility, in $94 \%$ of them tubes were found to be blocked. Therefore it is unnecessary to check them by HSG and they are candidates for IVF after medical treatment.

\section{Serious Consequences of Bacterial Vaginosis}

Dinsmoor, MJ. Associate Professor, Department of Obstetrics and Gynecology. Medical College of Virginia of Vir- ginia Commonwealth University. Richmond, Virginia; USA Bacterial vaginosis (BV), an imbalance in the vaginal ecosystem, is the most commonly diagnosed vaginal "infection" in the United States, although up to $50 \%$ of patients may be asymptomatic. It is important to screen and treat for this condition, as it has been associated with a number of adverse outcomes.

In nonpregnant women, those with BV frequently have evidence of upper genital tract disease, including histologic evidence of endometritis. BV organisms are isolated in most cases of pelvic inflammatory disease, and patients with BV have an increased incidence of postabortal infection and of cuff cellulitis following hysterectomy. BV has also been associated with an increased risk of cervical dysplasia. Trials to study the efficacy of treatment in preventing these complications are lacking.

In the pregnant patient, the presence of $\mathrm{BV}$ or BV organisms has been linked to an increased risk of infectious complications, including intra-amniotic infection and postpartum endometritis. BV organisms are frequently isolated in these peripartum infections. The risk of preterm birth (PTB) is also increased 3 to 7 fold in the presence of BV. Treatment trials in pregnant women reveal that systemic treatment of BV decreases the risk of PTB in women who are at high risk for this complication. The benefit of BV treatment in women at low risk for PTB is less clear, as is the impact on the incidence of infectious complications.

In conclusion, BV is a common condition with myriad consequences. Screening for and treatment of bacterial vaginosis is an important part of gynecologic and obstetric care.

\section{Genital Mycoplasmas \\ Aydin D}

Department of Microbiology and Clinical Microbiology, Medical Faculty of Istanbul, Çapa-Istanbul, Turkey

Mycoplasmas are the smallest free-living organisms. They lack a rigid cell wall that renders them poorly stainable by Gram method, resistant to cell wall-acting antimicrobials (such as penicillins, cephalosporins, etc.) and display extreme pleomorphism.

Of the mycoplasmal species that can be encountered in the normal human genital tract, Ureaplasma urealyticum, $M y$ coplasma hominis, and Mycoplasma genitalium are considered important.

Ureaplasma urealyticum and $M$ hominis can be isolated from healthy persons in high percentages; $40-50 \%$ of healthy females and $30-40 \%$ of healthy men include these microorganisms as part of their resident genital flora.

Genital mycoplasmas constitute a smaller part of all female genital tract infections than is supposed. The association of genital mycoplasmas with female genital tract are categorized in three groups; cases in which genital mycoplasmas are proved to be as definitive causative agents, cases in which they are deemed to be probable causative agents, and cases in which it is shown that they are not the causative agents.

Although there are considerable findings that $U$. urealyticum may play a role in repeated spontaneous abortion, stillbirth and Reiter syndrome, and $M$. hominis in abscess of Bartholin's gland, further studies are still needed to ascertain these findings.

Genital mycoplasmas are not causes of vaginitis and cervicitis. 
The most reliable and frequently used methods for diagnosis are culture for U. urealyticum and M. hominis, and PCR for $M$. genitalium.

Doxycycline is the preferred drug in the treatment of NGU. In cases that are resistant to tetracyclines, macrolides or quinolones may be used.

\section{The ABC's of Hepatitis in Pregnancy}

Dinsmoor, MJ. Associate Professor, Department of Obstetrics and Gynecology. Medical College of Virginia of Virginia Commonwealth University. Richmond, Virginia; USA Viral hepatitis is a frequent complication of pregnancy, usually in its chronic form. A review of the different types of viral hepatitis follows.

The incidence and prognosis of hepatitis $\mathrm{A}$ is not different in pregnancy. It is not teratogenic, and is very rarely vertically transmitted. A vaccine, which is safe in pregnancy, is currently available.

Most pregnant patients with hepatitis $B$ are chronic carriers. Since most seropositive pregnant women have no risk factors for infection, routine screening for this infection is recommended for all pregnant women in the United States. Babies of women with hepatitis B are given HBIG and hepatitis $B$ vaccine at the time of birth, while babies of seronegative women are administered the vaccine alone. Breast feeding does not increase the risk of vertical transmission.

The most seroprevalent form of viral hepatitis in pregnancy is hepatitis $\mathrm{C}$, although $25-50 \%$ of infected women have no risk factors for infection. The risk of vertical transmission is not high (probably $<10 \%$ ), although it is increased if the mother is also HIV-infected or has circulating HCV RNA. The long term neonatal outcome following perinatal hepatitis $\mathrm{C}$ infection is not known. Breast feeding does not appear to increase the risk of vertical transmission, although scant data are available.

Hepatitis D is present only in conjunction with acute or chronic hepatitis B infection. Measures to prevent spread of hepatitis B will also be effective in curtailing hepatitis D. Hepatitis E is usually diagnosed on clinical grounds, and causes a mild and self-limited illness in most cases. In developing countries, there appears to be a higher attack rate and higher case-fatality rate during pregnancy. Vertical transmission can occur, but it is not known how frequently.

\section{Vaccination in Pregnancy}

Dokuzoiuz, B.

Ankara Numune Research and Training Hospital Dept. of Infectious Diseases and Clinical Microbiology, Ankara, Turkey

As people realized that childhood vaccine program is insufficient to eliminate some vaccine preventable diseases, adolescent and adult vaccine scheme were found to be important. On the other hand, the beginning of childbearing age of women was accepted as about 15 . Thus, the period of pregnancy and routine vaccination program may coincide. Vaccination of woman whose pregnancy is not known may cause serious problems. Also, pregnant women may need vaccination for epidemics, travel, occupational and environmental exposure, etc. Because of unknown but theoretical risk to the fetus, immunization of pregnant women is generally avoided. Tetanus vaccine has a special importance. To avoid puerperal and neonatal tetanus, all the pregnant women nonor inadequately immunized before have to be vaccinated by tetanus vaccine. Inactivated, recombinant, polysaccharide vaccines(hepatitis $B$, hepatitis $A$, influenzae, meningococcal vaccine, pneumococcal vaccine, rabies, inactivated polio vaccine) can be administered safely to pregnant women if there is a necessity. However, live vaccines (oral polio vaccine, measles, mumps, rubella, yellow fever, varicella vaccine) are certainly contraindicated for pregnant women. Although, oral polio vaccine and yellow fever vaccine are live vaccines, they may be applied to pregnant women in very exceptional situations. Immune globulins are safe for pregnant women.

Continued Decreased Vertical Transmission With Combination Antiretroviral Therapy in Pregnancy

Hammill,. Hunter A; Shearer W; Hanson C; Polk R.: Baylor College of Medicine, Houston, Texas USA

Objectives: To review our experience with regard to maternal safety and infant outcome in HIV-infected women treated with triple combination therapy during gestation.

Design: Retrospective, single-center study.

Methods: A chart review is being conducted of women who initiated triple therapy with combivir and nelfinavir or combivir and indinavir usually at 20 weeks gestation and who have delivered their infants. Virologic follow-up data on infant status is obtained from the Baylor College of Medicine Pediatric Department of Allergy and Immunology, as it becomes available.

Results: Data on 98 women who delivered between 21 July 97 and 1 August 1999 are summarized. Treatment was well tolerated; there were no unusual symptoms reported, and no increased frequency of symptoms normally seen in this patient population. Early nausea was felt to be due to pregnancy and not drugs. All patients received intrapartum zidovudine, in cases prior to membrane rupture. Eighty-four (84) deliveries were vaginal, including delivery of 6 sets of twins; and fourteen (14) by cesarean section including one premature delivery of 29 weeks due to chronic hypertension and severe pregnancy-induced hypertension. Ninety-six patients had viral load determinations done during gestation at their initial prenatal visit, ranging between undetectable (Digne $<25$ copies $/ \mathrm{ml}$ ) and 189,923 copies $/ \mathrm{ml}$. All patients had CD4 levels done during gestation, ranging between 8 and 1,107 cells/ $\mathrm{mm}^{3}$; CD4 levels at delivery were done on patients and ranged between 11 and 964 cells $/ \mathrm{mm}^{3}$. No fetal anomalies were observed at birth, suggesting that they will likely prove to be uninfected. Updated virologic data on infants delivered to date as well as clinical results for additional deliveries will be presented. At this time 1 infant is positive.

Conclusions: Triple combination therapy including combivir and nelfinavir or combivir and indinavir are well tolerated during pregnancy and was not associated with any fetal abnormalities in the small cohort described here. The transmission rate is less than $2 \%$ with combination therapy at the time of this abstract.

\section{Cervicovaginal Microbiology, Enzymes, and Preterm Labor}

De Domenico P., Coata G., Pennacchi L., Taddei F., Gerli S., Di Renzo G.C. Centre of Perinatal Medicine, University of Perugia, Perugia, Italy 
Introduction: Lower reproductive tract infections, such as cervicitis or vaginitis, may play a major role in the pathogenesis of preterm labor, premature rupture of membranes and preterm birth.

Objective: To investigate the relationship between cervicovaginal infections and related events as assessed by expression of bacterial enzymes and alterations in vaginal $\mathrm{pH}$ with preterm labor.

Materials and Methods: We identified cervicovaginal infections using three different diagnostic tools: 1) cervicovaginal microbiology in 1830 patients; 2 ) assessment of vaginal $\mathrm{pH}$ in 100 patients; 3 ) detection of bacterial enzymes in 50 patients. Then we correlated these events to perinatal outcome. We evaluated women in pregnancy at first trimester and during gestation week 30-34. Vaginal fluid was taken for wet mount, Gram stain, $\mathrm{pH}$ measurement, bacterial enzymes such as sialidase, and microbiological test. Endocervical swab was sampled by using a cotton-tipped swab. Vaginal $\mathrm{pH}$ values were obtained by colorimetric strips carried by index finger of the special gloves. A cut-off point of $\mathrm{pH}$ 4.5 was selected.

Results: In 1830 patients who have undergone microbiological test, we found $30 \%$ microbiological positivity. Arnong these, $52 \%$ developed threatening preterm labor. In $100 \mathrm{pa-}$ tients we detected a pH greater than 4.5 in $35 \%$ of all cases. Among microbiologically-positive patients (40\%), vaginal fluid $\mathrm{pH}$ was positive especially in $74 \%$ cases of BV. Sialidase activity in cervicovaginal fluid was associated with presence of ureaplasma, GBS, gardnerella and chlamydia.

Conclusion: Screening and treating cervicovaginal infections may be the best way to prevent adverse perinatal outcome such as preterm labor, PROM and preterm birth.

Chlamydia Infection and Perinatal Outcome

De Domenico P., Sensini A., Nicoli S., Cenci F., Di Renzo G.C. Centre of Perinatal Medicine, University of Perugia, Perugia, Italy

Objective: We evaluated the importance of Chlamydia trachomatis infection in the etiopathogenesis of adverse perinatal outcome such as preterm labor, premature rupture of membranes, and preterm birth.

Materials and Methods: At first trimester and during gestation week $30-34$, specimens were collected by inserting a sterile speculum into the vagina and wiping the vaginal discharge from the ectocervix. Endocervical material was sampled by using a cotton-tipped swab.

Results: The $C$. trachomatis infection rate was $8.8 \%$. PROM occurred in $35 \%$ of the cases; the frequency of threatening preterm labor was $10 \%$, significantly higher in C. trachomatis-positive patients.

Conclusion: Every pregnant woman, and not only those at high risk, should be screened for $C$. trachomatis infection in order to prevent adverse perinatal outcome.

Inflammatory Reactions in Cervico-Vaginal Smears Tanzer F, Saraçoilu OF

Terminal Medical Center, Ankara, Turkey

Aim: To investigate the rate of inflammation in cervico-vaginal smears taken from asymptomatic women to screen against cervical cancer.

Methods: 390 cervico-vaginal smears taken from women without any complaints in 1998 were studied for inflammatory reactions in Terminal Medical Center. All cervico-vaginal smears prepared from cells removed from the posterior fornix of the vagina or from the squamous epithelium that lines the cervix and the portio of the cervix from the epithelial lining of the endocervical canal. Cervico-vaginal smear of collection of a vaginal pool and cervical surface sampled by brush. They were fixed by spray fixative. All smears were stained by Papanicolau staining technique. Pathological inflammatory processes within the lower genital tract are bacterial, fungal, parasitic or viral origin.

Results: 238 (61.1\%) cervico-vaginal smears showed normal cytologic findings. Inflammatory reactions were found in $152(38.9 \%)$ of them. The cocci are the most common causative bacterial agents and found in 103 women $(26.4 \%)$. Candida was the most common fungus associated with vaginitis and cervicitis found in 17 cases (4.3\%). Trichomonas vaginalis was by far the most significant parasite found in 10 cases $(2.6 \%)$. Gardneralla was found in 11 cases $(2.8 \%)$. Viral agents herpes genitalis and human papilloma virus infections were the most important and found in 11 cases $(2.8 \%)$.

Conclusion: The cervicovaginal smears taken from asymptomatic women are useful to diagnose inflamations in asymptomatic cases or early infections.

\section{Perinatal Group B Streptococcal Colonization and Intra-} partum Antibiotic Prophylaxis

I. Akman M.D., G. Imir M.D., C. Dökmeci M.D., H. Bilgen M.D., M. Oral M.D., E. Özek M.D., N. Ceyhan M.D.

Marmara University, Faculty of Medicine, Dept. of Obstetrics and Gynecology

And Pediatrics, Istanbul, Turkey

Objective: Group B Streptococcus (GBS) is a common cause of serious maternal and neonatal infections. The aim of this study was to identify the incidence of rectovaginal colonization of GBS in pregnant women in our hospital and to evaluate the outcome of culture positive patients who had received intrapartum antibiotic prophylaxis according to recommendations of American Academy of Pediatrics.

Methods: Pregnant women followed at Marmara University Hospital, Obstetrics and Gynecology outpatient clinic were screened at week 35-37 of gestation for GBS colonization. The rectovaginal samples were processed in a selective culture medium for GBS. The patients with positive culture results received intravenous ampicillin in the intrapartum period and both the patients and their newborns were evaluated for complications.

Results: Out of 100 patients between $18-41$ years of age, 57 patients $(57 \%)$ were multipara and 43 patients $(43 \%)$ were primipara. Ten patients (10\%) (6 patients multipara, 4 patients primipara) had BGS growth in their rectovaginal cultures whereas 1 patient had urinary tract infection secondary to GBS. The multipara patients had not had any infectious complications in their previous pregnancies. All but two patients with GBS colonization received intrapartum antibiotic prophylaxis as well as treatment of urinary tract infection when present. One of the GBS $(+)$ patients had emergency cesarean section delivery for fetal distress and could not receive intrapartum antibiotic prophylaxis. The culture result of the other patient was not known during the labor so she could not receive intrapartum antibiotic prophylaxis as well. 
None of the patients developed any infections or had any side effects secondary to antibiotics used for prophylaxis. However, the newborn of the GBS $(+)$ patient who could not receive intrapartum antibiotic prophylaxis developed sepsis. Conclusion: The incidence of rectovaginal GBS colonization was found to be $10 \%$ in pregnant women followed at our hospital. Routine screening for GBS colonization at week 35-37 of gestation and using the intrapartum antibiotic prophylaxis might decrease the incidence of perinatal GBS infection.

Intrapartum Prophylaxis for Perinatal Group B Streptococcal Disease: Do the CDC Guidelines Apply Everywhere: The Rambam Experience

Peter Jakobi, M.D., Orly Goldstick, M.D., Polo Sujov, M.D., Joseph Itskovitz-Eldor, M.D., DSC

Objective: To establish whether to adopt the new guidelines from the CDC for the prevention of perinatal group B streptococcal disease in our region.

Design: A retrospective chart review of all neonates born during 1993-1997, with group B streptococcal colonization at any culture site. All rectovaginal cultures taken from group B streptococcus in parturients with preterm premature rupture of membranes and preterm labor during 1993-1997 were reviewed.

Results: During the 10 study years, 45,968 neonates were evaluated, 12 neonates developed early onset group B streptococcal sepsis $(0.26 / 1000)$. 8 neonates with a positive culture of group B streptococcus died, but only 2 of them had septicemia. 6 deaths, including the 2 with septicemia, could have been attributed to other factors. During the years 19931997, rectovaginal cultures unselective media were sent for group B streptococcus, for 259 parturients with preterm premature rupture of membranes or preterm labor. Only $18(7 \%)$ were positive, none of their babies developed early onset group B streptococcal sepsis.

Conclusion: The low carrier rate in our high risk patients and the low incidence of neonatal early onset group B streptococcal disease in our population lead us to reject the new guidelines from the CDC.

Sexually Transmitted Diseases Incidence In Entertainment Business In Ankara

Karaiahin E., Saraçoilu F., Balaban E., Güvener E.

Ankara Numune Hospital Dept. of Gynecology and Obstetrics, and Dept. of Microbiology

Objective: This survey has been planned to investigate the foreign (mainly Russian and Romanian) and local women in entertainment business, as possible foci of gonorrhea, syphillis, hepatitis B \& C, HIV, as well as trichomoniasis, candidiasis, bacterial vaginosis, ureaplasmosis and mycoplasmosis.

Method: 74 women were sampled bacteriologically and serologically for the mentioned sexually transmitted disesases and have been asked to fill out a questionnaire.

Results: No cases of chlamydia, trichomonas, HIV, and hepatitis $\mathrm{C}$ were identified. The incidences of other infections were found as: candidiasis $(5.4 \%)$, bacterial vaginosis $(12.16 \%)$, ureaplasmosis $(21.6 \%)$, mycoplasmosis $(16.2 \%)$, gonorrhea (5.4\%), syphillis $(1.35 \%)$, and hepatitis $\mathrm{B}(2.7 \%)$.

Conclusion: There has been an insidious increase in STD in Turkish population as stated and supported by various other researches. We tried to open a less clearly visible source of STD and highlighted some of the unknown risk factors present in entertainment business in Ankara region.

Evaluation of 62 Tubo-Ovarian Abscesses: Risk Factors, Clinical Features, and Treatment

Atalay C., Saraçoilu F., Uyar I.

Gynecology and Obstetric Department of Ankara Numune Education and Research Hospital

Objective: Evaluation of risk factors, clinical features and treatment in tubo-ovarian abscess which is one of the early complications of pelvic inflammatory disease with a prevalance as many as $34 \%$ of women with PID.

Material and Methods: 62 patients who were treated at the Obstetrics and Gynecology Department of Ankara Numune Education and Research Hospital between 1995 and 1999 were evaluated, retrospectively.

Results: The patients ranged in age between and 20-54 with a mean of $36.16 \pm 8.36$. All of the patients were married. The parities were between $0-5$ with a mean of $2.06 \pm 1.54$.

In our study. the most important risk factor was IUD usage $(58.06 \%)$. The most frequent symptom was abdominal pain (90.3), the other symptoms were foul smelling vaginal discharge $(69.3 \%)$ and vaginal bleeding $(29.03 \%)$. In pelvic examination bilateral adnexial masses were found in $16(25.8 \%)$ of the patients, left adnexial mass was found in $28(45.16 \%)$, and right adnexial mass was found in $18(29.03 \%)$ of the patients. The fever was more than $38^{\circ} \mathrm{C}$ in $38(61.3 \%)$ patients. The mean leucocyte count was $15.793 \pm 5032.75$. There was a correlation with ultrasound examination in 40 of $62(64.5 \%)$ patients. Clindamycin and gentamicin treatment was performed to all patients. After 48 hours 17(27.4\%) patients responded to medical treatment. The other $45(72.5 \%)$ patients were treated with surgical methods. There was $25.8 \%$ rupture and $35.4 \%$ generalized peritonitis. In our cases we had no complications, mean duration of hospitalization was 11.03 \pm 5.1 days.

\section{Acute Appendicitis in Pregnancy}

Atalay C., Saraçoilu F., Ekici A.

Gynecology Department of Ankara Numune Research and Education Hospital

Objective: Acute appendicitis in pregnancy is the most common nonobstetric complication warranting emergency laparotomy. The goal of this study is the evaluation of clinical manifestations, diagnosis and management of acute appendicitis in pregnancy.

Material and Methods: 25 pregnant patients to whom performed laparotomy for suspected acute appendicitis in Gynecology Department of Ankara Numune Research and Education Hospital between 1995-1999 were evaluated retrospectively.

Results: In $15(60 \%)$ cases, acute appendicitis was proven at laparotomy and by subsequent histopathology. In the confirmed cases, $3(25 \%)$ in the first trimester, $18(72 \%)$ in the second and $4(16 \%)$ in the third trimester. Abdominal pain was the most common presenting symptom; the second was vomiting.Abdominal tenderness and rebound tenderness were the most common physical signs, although the latter was less marked in late pregnancy. There was a correlation with USG in $2(8 \%)$ patients. Laparotomy was performed within the 
first 24 hours of onset of symptoms in all patients. Perforation of appendix was found in $1(4 \%)$ patient. There were $2(8 \%)$ preterm labors in the third trimester group (30 and 34 weeks) within the postoperative 48 hours, tocolitic therapy was performed and successful. In our study, there were no fetal and maternal deaths.

\section{How Should We Treat Bacterial Vaginosis in the 1990s?} Larsson, P-G.

Departments of Obstetrics and Gynecology Karnsjukhuset, Skovde, Sweden.

The treatment of anaerobic/bacterial vaginosis can sometimes be difficult. To calculate the true four-week cure rate, endpoint analysis should be carried out. The patients not cured after one or two weeks should be added to patients not cured after four weeks (many authors exclude patients not cured at one or two weeks). After a critical review of the literature of all blinded (single or double) studies the cure rate is much lower than expected and is no better than $60-75 \%$.

An analysis shows that nonpublished treatment studies (used by the drug companies in registration files) have a much lower cure rate than the published studies. If the expected cure rate after 4-weeks is so low, how shall we increase the cure rate?
Women visiting for legal abortions will have benefit of preoperative treatment of $\mathrm{BV}$ with vaginal clindamycin cream the same as women with intermediate flora. However, women with normal lactobacilli will not have any increased risk of postoperative infection. This will point out that maybe even intermediate flora should be regarded as a pathological flora and should be regarded as pathological as BV and should be treated the same.

Perinatal Group B Streptococcal Colonization and Intrapartum Antibiotic Prophylaxis

I. Akman M.D., G. Imir M.D., C. Dökmeci M.D., H. Bilgen M.D., M. Oral M.D., E. Özek M.D., N. Ceyhan M.D.

Marmara University, Faculty of Medicine, Dept. of Obstetrics \& Gynecology and Pediatrics, Istanbul, Turkey

\section{Group B Streptococcus: 1999 Update}

Dinsmoor, MJ. Associate Professor, Department of Obstetrics and Gynecology. Medical College of Virginia of Virginia Commonwealth University. Richmond, Virginia; USA 


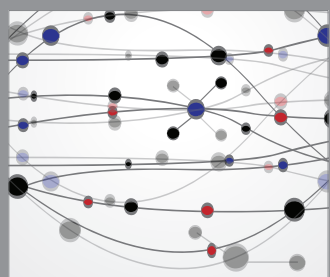

The Scientific World Journal
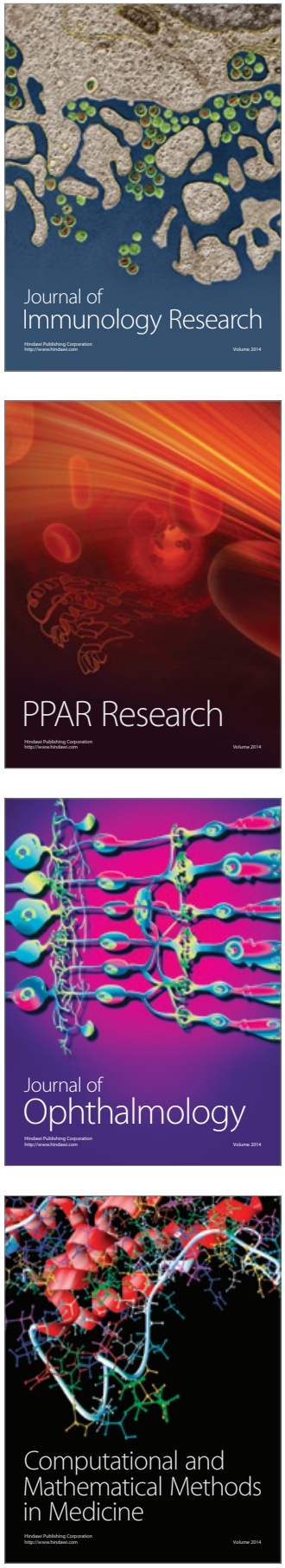

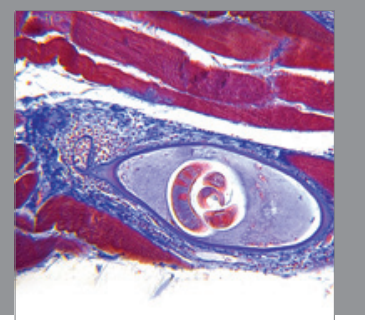

Gastroenterology

Research and Practice
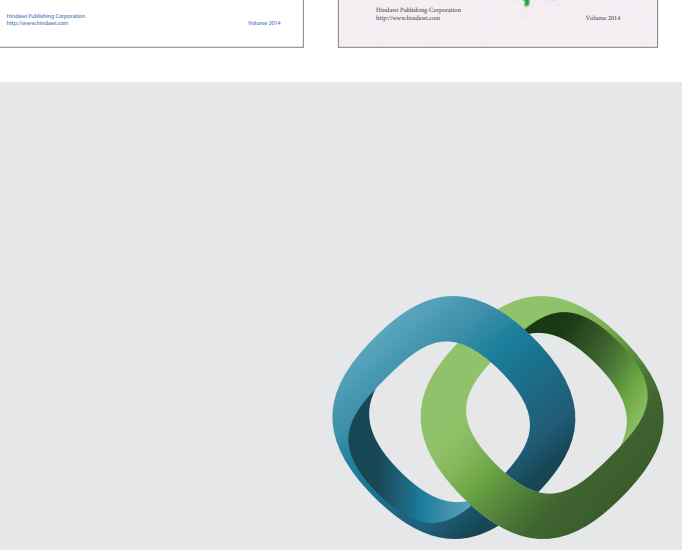

\section{Hindawi}

Submit your manuscripts at

http://www.hindawi.com
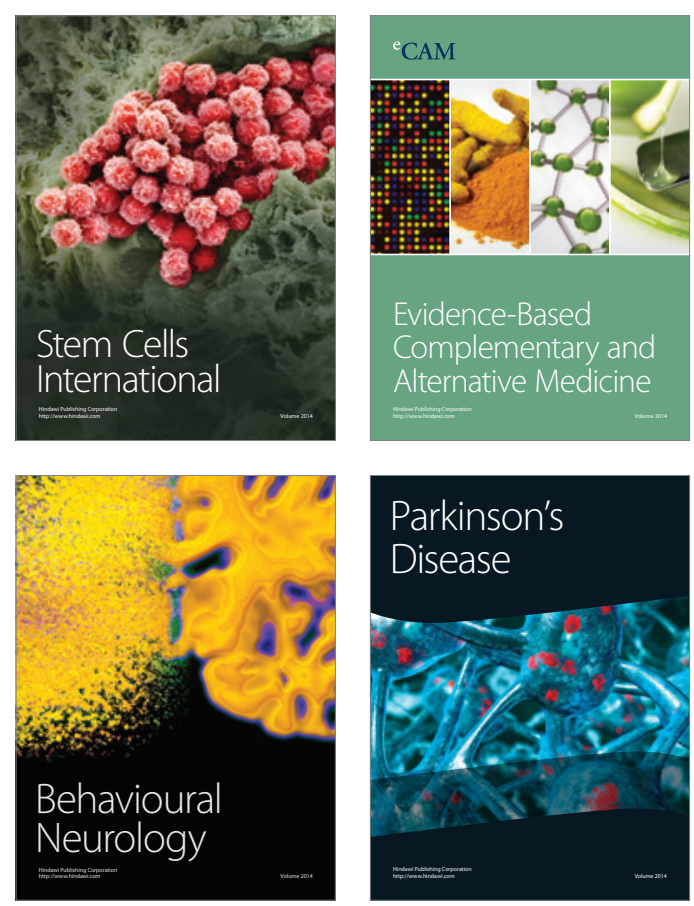

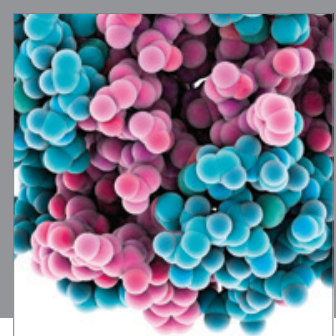

Journal of
Diabetes Research

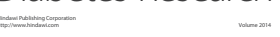

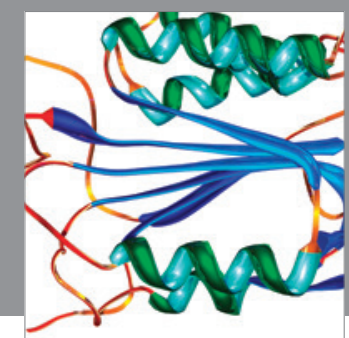

Disease Markers
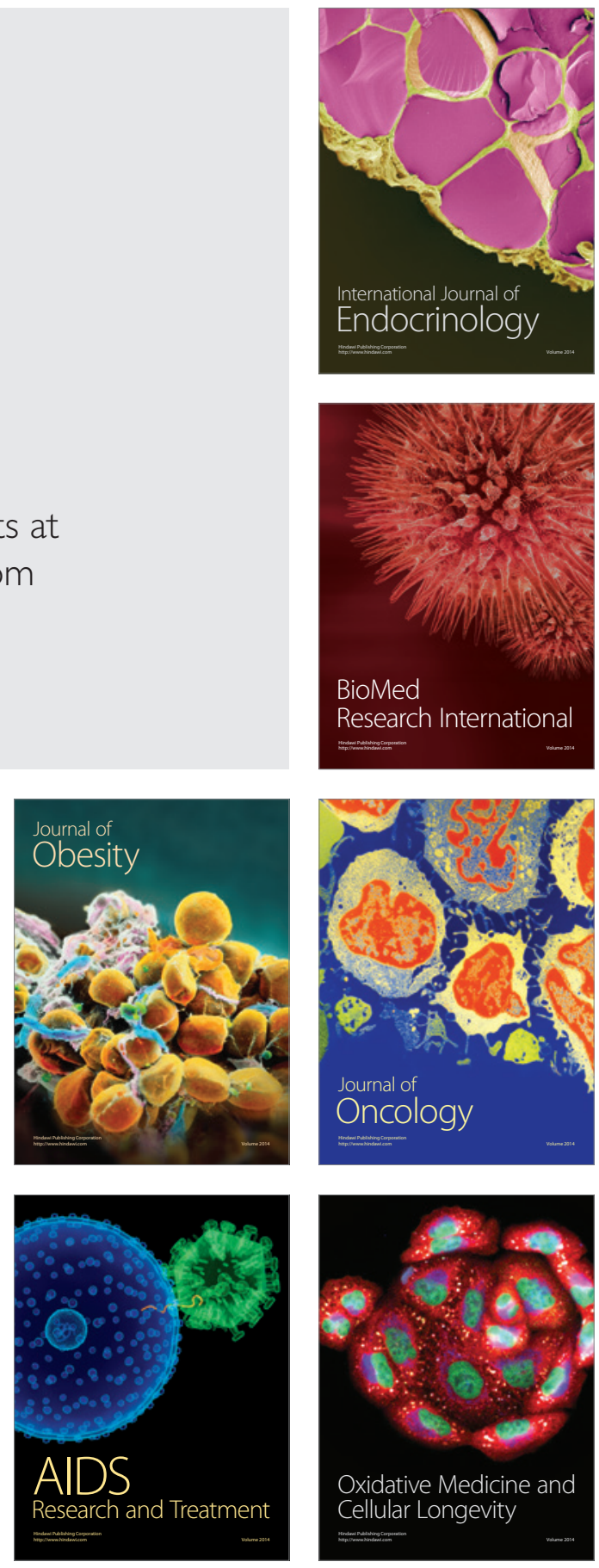